\title{
Nonthermal leptogenesis and UV freeze-in of dark matter: Impact of inflationary reheating
}

\author{
Basabendu Barman $\oplus^{*}$ Debasish Borah $\odot,{ }^{\dagger}$ and Rishav Roshan $\odot^{\ddagger}$ \\ Department of Physics, Indian Institute of Technology Guwahati, Assam 781039, India
}

(Received 18 May 2021; accepted 22 July 2021; published 23 August 2021)

\begin{abstract}
We study a minimal scenario to realize nonthermal leptogenesis and UV freeze-in of a Standard Model (SM) gauge singlet fermionic dark matter (DM) simultaneously, with inflaton field playing a nontrivial role in their yields. The renormalizable interactions are restricted to the SM fields, two right-handed neutrinos (RHN) and inflaton coupling exclusively to the RHNs, while the DM couples to both the SM and the RHNs only via operators of dimension $d>4$. Considering two separate cases of $d=\{5,6\}$, we show that for $d=5$, inflaton decay into RHNs followed by their subsequent decay into SM particles lead to both reheating as well as DM production from the SM bath. This requires a cut off scale as large as $\Lambda \sim$ $10^{17} \mathrm{GeV}$ depending on the DM mass. On the other hand, for $d=6$, DM production happens directly from scattering of RHNs (for $\Lambda \gtrsim 10^{14} \mathrm{GeV}$ ) that results in a very nontrivial evolution of the DM yield. In both these cases, it is possible to explain the observed baryon asymmetry through successful nonthermal leptogenesis via the decay of the RHNs, together with the Planck observed relic density of the DM via pure UV freeze-in mechanism. Taking into account both instantaneous as well as noninstantaneous reheating separately, we constrain the parameter space of this minimal scenario from relevant phenomenological requirements including sub-eV scale active neutrino masses and their mixing.
\end{abstract}

DOI: $10.1103 /$ PhysRevD.104.035022

\section{INTRODUCTION}

The observed matter-antimatter asymmetry of the Universe has been a longstanding puzzle [1,2]. While approximately $5 \%$ of our present Universe is dominated by visible matter, most of it is made up of baryons. Quantitatively, the asymmetry is quoted as a ratio of excess of baryons over antibaryons to photons. According to the latest data from the Planck satellite it is given as [2]

$$
\eta_{B}=\frac{n_{B}-n_{\bar{B}}}{n_{\gamma}}=6.1 \times 10^{-10} .
$$

The estimate from the cosmic microwave background (CMB) measurements also matches very well with the ones obtained from big bang nucleosynthesis (BBN). Since the Universe is expected to originate in a matter-antimatter symmetric manner and any preexisting initial asymmetry is likely to be diluted due to accelerated expansion during

\footnotetext{
*bb1988@iitg.ac.in

†borah@iitg.ac.in

rishav.roshan@iitg.ac.in
}

Published by the American Physical Society under the terms of the Creative Commons Attribution 4.0 International license. Further distribution of this work must maintain attribution to the author(s) and the published article's title, journal citation, and DOI. Funded by SCOAP ${ }^{3}$. inflation, there arises the requirement of a dynamical explanation for the observed asymmetry. Such a dynamical process is required to satisfy certain conditions, given by Sakharov [3] as (1) baryon number (B) violation, (2) charge-conjugation $(\mathrm{C})$ and charge-parity $(C P)$ violation, and (3) departure from thermal equilibrium, all of which cannot be satisfied in the required amount in the standard model (SM) of particle physics and considering an expanding Friedmann-Lemaitre-Robertson-Walker (FLRW) Universe. Out-of-equilibrium decay of a heavy particle leading to the generation of baryon asymmetry of the Universe (BAU) has been a well-known mechanism for baryogenesis $[4,5]$. One interesting way to implement such a mechanism is leptogenesis [6], where a net leptonic asymmetry is generated first which then gets converted into baryon asymmetry through $(B+L)$-violating electroweak (EW) sphaleron transitions [7]. For the lepton asymmetry to be converted into baryon asymmetry, it is important that the processes giving rise to the leptonic asymmetry freeze-out before the onset of the sphaleron transitions to prevent wash out of the asymmetry [8]. An interesting feature of this scenario is that the required lepton asymmetry can be generated through $C P$ violating out-of-equilibrium decays of the same heavy fields that take part in the seesaw mechanism [9-15] that explains the origin of tiny neutrino masses [1], another observed phenomenon the SM fails to address. In such generic seesaw models for light neutrino 
masses, the scale of leptogenesis remains very high. For example, in the Type-I seesaw framework, the requirement of producing the correct lepton asymmetry pushes the scale of right-handed neutrinos to a very high scale $(M>$ $10^{9} \mathrm{GeV}$ ) known as the Davidson-Ibarra bound [16] of high scale or vanilla leptogenesis. ${ }^{1}$ If the temperature of the Universe can reach a value equal or above the mass of righthanded neutrinos, they can be thermally produced from the bath particles. However, the reheat temperature of the Universe after inflation $\left(T_{\mathrm{RH}}\right)$ can, in principle, be as low as a few $\mathrm{MeV}$; the typical temperature around the BBN epoch. Therefore, a low reheat temperature will forbid the production of right-handed neutrinos in the thermal bath. However, they can still be produced nonthermally from the inflaton field, leading to the scenario of nonthermal leptogenesis [19-36].

Similar to matter-antimatter asymmetry, observations of a mysterious nonluminous, nonbaryonic form of matter, known as dark matter (DM) in the Universe has been another puzzle. Cosmology based experiments like Planck [2], through precise measurements of cosmic microwave background anisotropies, suggest around 26\% of the present Universe's energy density to be made up of DM. In terms of density parameter $\Omega_{\mathrm{DM}}$ and $h=$ Hubble Parameter $/\left(100 \mathrm{kms}^{-1} \mathrm{Mpc}^{-1}\right)$, the present $\mathrm{DM}$ abundance is conventionally reported as [2]; $\Omega_{\mathrm{DM}} h^{2}=$ $0.120 \pm 0.001$ at $68 \%$ C.L. Astrophysics related observations have also been suggesting the same for a much longer period [37-39]. While none of the SM particles can be a viable DM candidate, several beyond the standard model (BSM) proposals have been studied out of which the weakly interacting massive particle (WIMP) paradigm is the most popular one. The WIMP paradigm relies upon weak type-interactions between the DM and SM sectors which not only play crucial role in generating its relic abundance from thermal freeze-out [40-42] but can also lead to observable DM-nucleon scattering rates at direct detection experiments. However, null results at such direct detection experiments like XENON1T [43] have led to growing interest in alternative DM formalisms in recent times. One such popular alternative is the feebly interacting massive particle (FIMP) scenario where DM interaction with the SM bath is so weak that it never attains thermal equilibrium at any epoch in the early Universe $[44,45]$. The initial abundance of such a particle is considered to be negligible and it can start populating (or freeze-in) the Universe due to decay or annihilation of other particles in the thermal bath. If there exists renormalizable DM-SM coupling in the FIMP scenario, the nonthermal criterion

\footnotetext{
${ }^{1}$ For right-handed neutrino masses lower than the DavidsonIbarra bound, say around $\mathrm{TeV}$ scale, it is still possible to generate correct lepton asymmetry by resorting to a resonant enhancement of the $C P$-asymmetry with a quasidegenerate right-handed neutrino spectrum $[17,18]$, known as resonant leptogenesis.
}

enforces the couplings to be extremely tiny via the following condition $\left|\frac{\Gamma}{\mathbf{H}}\right|_{T \simeq M_{0}}<1$ [46], where $\Gamma$ is the decay width. For the case of scattering, one has to replace $\Gamma$ by the interaction rate $n_{\mathrm{eq}}\langle\sigma \mathrm{v}\rangle$, where $n_{\mathrm{eq}}$ is the equilibrium number density of the mother particle. These types of freeze-in scenarios are known as infrared (IR)freeze-in [45,47-67] where DM production is dominated by the lowest possible temperature at which it can occur i.e., $T \sim M_{0}$, since for $T<M_{0}$, the number density of mother particle becomes Boltzmann suppressed. Another possibility to understand feeble DM-SM interaction is to consider higher-dimensional operators (dimension $d>4$ ) connecting DM and SM sectors. Unlike the IR freeze-in scenario, here the DM production is effective at high temperatures and relies upon the details of reheating after the end of inflation. Since DM-SM operators are of dimension five or above, typically, DM is produced via scattering specially at temperatures above the electroweak scale. This particular scenario is known as the ultra-violet (UV) freeze-in [44,68-77].

Motivated by these, here we study a minimal scenario where both nonthermal leptogenesis and UV freeze-in of DM can be realized simultaneously. While two singlet right-handed neutrinos (RHN) as in Type-I seesaw framework $^{2}$ take care of leptogenesis, a $Z_{2}$ odd SM gauge singlet fermion is introduced as DM which interacts with the SM along with the RHNs only via operators of dimension $d>4$, which is necessary for UV freeze-in. Considering the inflaton to be a singlet scalar, we assume it is renormalizable coupling with the RHNs alone so that the inflaton decays into a pair of RHNs followed by the latter's out-of-equilibrium decay into the SM particles leading to reheating. We consider both dimension five and dimension six operators separately to study the UV freeze-in of DM while simultaneously solving the coupled Boltzmann equations for nonthermal leptogenesis and reheating. Depending upon inflaton coupling with the RHNs, we outline two cases: instantaneous reheating (where the inflation energy density is converted into the heavy neutrinos instantaneously) as well as noninstantaneous (where the inflation energy density is converted into the heavy neutrinos over some finite time) reheating, where the corresponding evolution of DM and lepton asymmetry are determined with a suitable choice of parameters and initial conditions. Without going into the details of the dynamics of inflation, in the present scenario we look into the post slow-roll era when the inflaton energy starts converting into the energy of the heavy neutrinos. See [78] for similar studies separating the period of inflation from reheating and references therein for specific models of inflation which allow this possibility. This separation also justifies the fact

\footnotetext{
${ }^{2}$ Even though three RHNs are typically introduced in Type-I seesaw, two are sufficient to generate observed neutrino oscillation data with vanishing lightest neutrino mass.
} 
that we have a cut off scale in the theory which is lower than the Planck scale while inflationary field excursions can, in general, be super-Planckian. We typically probe the $T_{\mathrm{RH}} \lesssim M_{\varphi}$ scenario, where $M_{\varphi}$ is the inflaton mass and $T_{\mathrm{RH}}$ is the reheating temperature. This is in contrast to most of the cases where generally $M_{\varphi} \gg T_{\mathrm{RH}}$ is assumed. From the requirement of successful nonthermal leptogenesis leading to observed BAU (DM relics also the correct neutrino mass) we finally constrain the key parameters namely, the DM mass, reheat temperature, cut off scale $\Lambda$, as well as the inflaton coupling with RHNs. Also, for the dimension-six case we find that the DM abundance shows a very nontrivial evolution as it is produced only from the heavy right-handed neutrinos which are absent when it is produced from the radiation, as in the case of dimension five.

The paper is organized as follows. In Sec. II, we briefly discuss our framework followed by a discussion of nonthermal leptogenesis in Sec. III. In Sec. IV we elaborate the UV freeze-in setup for calculating the DM yield following the details of relevant coupled Boltzmann equations in Sec. V. Section VI summarizes our results for both instantaneous and noninstantaneous scenarios. In Sec. VII we briefly discuss possible UV completion of DM-SM operators and finally in Sec. VIII we conclude.

\section{THE FRAMEWORK}

We extend the Standard Model effective field theory (SMEFT) with two right-handed neutrinos $N_{R}$ which is commonly known as the $\nu$ SMEFT [79-81]. Assuming only SM gauge symmetries, the renormalizable interaction is given by the SM-Lagrangian with a sterile Majorana neutrino contribution as

$$
\begin{aligned}
\mathcal{L}_{\text {renorm }}= & \mathcal{L}_{\mathrm{SM}}+i \overline{N_{R}^{c}} \not \partial N_{R}-\left(\frac{1}{2} M_{N} \overline{N_{R}^{c}} N_{R}+\text { h.c }\right) \\
& +\left(-y_{N} \overline{\ell_{L}} \tilde{H} N_{R}+\text { H.c. }\right) .
\end{aligned}
$$

This Lagrangian is then extended by adding the dimension five (dim.5) and dimension six (dim.6) terms of the form

$$
\mathcal{L}_{\text {eff }}=\mathcal{L}_{\text {renorm }}+\frac{1}{\Lambda} \sum_{i} \mathcal{C}_{i} \mathcal{O}_{i}^{d=5}+\frac{1}{\Lambda^{2}} \sum_{i} \mathcal{C}_{i}^{\prime} \mathcal{O}_{i}^{d=6},
$$

where a basis of the operators $\mathcal{O}_{i}$ up to and including dim.6 will be elaborated on in Sec. II A and Sec. II B assuming CP conservation. We further consider a SM gauge singlet fermion $\chi$ odd under an imposed $Z_{2}$ symmetry that makes it absolutely stable over the age of the Universe and hence a potential dark matter (DM) candidate. Since it is a gauge singlet fermion (like the right-handed neutrinos) and $Z_{2}$-odd, it does not have any tree-level interaction either with the SM fields or with the RHNs. One can then write generalized DM-SM nonrenormalizable interactions as

$$
\mathcal{L} \supset \frac{c_{i j} \mathcal{O}_{\mathrm{SM}}^{(d) j} \mathcal{O}_{\mathrm{DM}}^{\left(d^{\prime}\right) i}}{\Lambda_{i j}^{d+d^{\prime}-4}}
$$

where $\mathcal{O}_{\mathrm{DM}}^{\left(d^{\prime}\right)}$ is a dark sector operator of mass dimension $d^{\prime}$, $\mathcal{O}_{\mathrm{SM}}^{(d)}$ is the operator in the visible sector of mass dimension $d$. The parameter $\Lambda_{i j}$ is a dimensionful scale and $c_{i j}$ is the dimensionless Wilson coefficient. If $d+d^{\prime}>4$, then the interaction is associated with an effective nonrenormalizable operator of the form presented in Eq. (4). All DM-SM interactions are encoded by higher-dimensional operators, with a cut off scale $\Lambda$, which is the mass scale of the heavy fields integrated out to obtain the low-energy Lagrangian.

Thus,

$$
\mathcal{L}=\mathcal{L}_{\mathrm{SM}}+\mathcal{L}_{\mathrm{DM}}+\mathcal{L}_{d>4},
$$

where $\mathcal{L}_{\mathrm{SM}(\mathrm{DM})}$ is the renormalizable $\mathrm{SM}(\mathrm{DM})$ Lagrangian and $\mathcal{L}_{d>4}$ corresponds to the operators of dimension $d>4$. In the renormalizable level the Lagrangian for the DM field has the form

$$
\mathcal{L}_{\mathrm{DM}}=i \overline{\chi^{c}} \not \partial \chi-\frac{1}{2} M_{\chi} \overline{\chi^{c}} \chi,
$$

as the DM is an electroweak singlet with zero hypercharge. Note that, the cut off scale in Eqs. (3) and (4) are the same implying that the low-energy theory containing the righthanded neutrinos and the DM is generated by integrating out all the heavy fields at a single scale $\Lambda$. For simplicity, we shall stick to scalar DM bilinears which show that at the level of dim.5, the DM production takes place from the thermal bath through the gauge-invariant interaction of the form [75]

$$
\mathcal{O}_{5}^{\mathrm{DM}-\mathrm{SM}}=\frac{1}{\Lambda} \bar{\chi} \chi H^{\dagger} H
$$

where $H$ is the SM Higgs doublet. The only source for the heavy neutrinos, on the other hand, is the on-shell decay of the inflaton which is assumed to couple exclusively to them. ${ }^{3}$ This also implies that the kinematical condition $M_{\varphi}>2 M_{N}$ always holds where $M_{\varphi}$ is the inflaton mass and $M_{N}$ is the mass of the heavy neutrino. In our case $M_{N} \equiv M_{1}$ which is the lightest heavy neutrino that is responsible for generating the required lepton asymmetry. As we shall argue, in the nonthermal leptogenesis setup we consider hierarchical right-handed neutrinos $M_{2} \gg M_{1}$

\footnotetext{
${ }^{3}$ Heavy right-handed neutrinos can also be produced from the SM fields by virtue of their Yukawa couplings if the highest temperature of the SM bath exceeds the mass of heavy neutrinos. However, this is not the case in nonthermal leptogenesis pursued here.
} 
which, in turn, also implies $2 M_{1}<M_{\varphi}<2 M_{2}$. Now, since we are considering an effective description, hence we consider $\Lambda$ to be the highest scale of the theory. For completeness, we write down the full list of operators up to and including dim.6 involving SM fields and right-handed neutrinos $N_{R}$ [79,81-84], along with the operators involving the DM and the SM fields plus $N_{R}$. However, as we shall discuss, for simplicity in the main analysis we will confine ourselves only to the scalar operators involving different fields.

\section{A. Dim.5 $\nu$ SMEFT operators}

At dim.5 the SMEFT contains only the Weinberg operator, while the RHN extension allows the additional term

$$
\mathcal{O}_{5}=i\left(H^{\dagger} H\right)\left(\overline{N_{R}^{c}} N_{R}\right)+\text { H.c. }
$$

\section{B. Dim.6 $\nu$ SMEFT operators}

In dim.6 the tree-generated (TG) operators can be classified on the basis of number of associated Higgs fields

$$
\begin{aligned}
\mathcal{O}_{6}^{2 h}\left[\psi^{2} \varphi^{2}\right]= & \left(H^{\dagger} i D^{\mu} H\right)\left(\overline{N_{R}^{c}} \Gamma_{\mu} N_{R}\right), \\
& \left(\overline{N_{R}^{c}} \gamma_{\mu} e_{R}\right)\left(\tilde{H}^{\dagger} i D^{\mu} H\right)+\text { H.c } \\
\mathcal{O}_{6}^{3 h}\left[\psi^{2} \varphi^{3}\right]= & \left(H^{\dagger} H\right)\left(\overline{\ell_{L}} \tilde{H} N_{R}\right)+\text { H.c. }
\end{aligned}
$$

The dim.6 operators involving 1-Higgs, e.g., $\left(\bar{L} \sigma^{\mu \nu} N_{R}\right) \tilde{H} B_{\mu \nu},\left(\bar{L} \sigma^{\mu \nu} N\right) \tau^{i} \tilde{H} W_{\mu \nu}^{i}$ are loop-generated (LG) operators and hence suppressed by $1 / 16 \pi^{2}$ [79]. There are also four-fermion contact terms in dim.6 level as follows:

$$
\begin{aligned}
\mathcal{O}_{6}^{L L R R}= & \left(\overline{\ell_{L}} \gamma_{\mu} \ell_{L}\right)\left(\overline{N_{R}^{c}} \Gamma^{\mu} N_{R}\right),\left(\overline{Q_{L}} \gamma_{\mu} Q_{L}\right)\left(\overline{N_{R}^{c}} \Gamma^{\mu} N_{R}\right) ; \\
\mathcal{O}_{6}^{R R R R}= & \left(\overline{e_{R}} \gamma_{\mu} e_{R}\right)\left(\overline{N_{R}^{c}} \Gamma^{\mu} N_{R}\right),\left(\overline{u_{R}} \gamma_{\mu} u_{R}\right)\left(\overline{N_{R}^{c}} \Gamma^{\mu} N_{R}\right), \\
& \left(\overline{d_{R}} \gamma_{\mu} d_{R}\right)\left(\overline{N_{R}^{c}} \Gamma^{\mu} N_{R}\right),\left(\overline{d_{R}} \gamma_{\mu} u_{R}\right)\left(\overline{N_{R}^{c}} \Gamma^{\mu} e\right)+\text { H.c.; } \\
\mathcal{O}_{6}^{L R L R}= & \left(\overline{\ell_{L}} N_{R}\right) \epsilon\left(\overline{\ell_{L}} e_{R}\right)+\text { H.c., } \\
& \left(\overline{\ell_{L}} N_{R}\right) \epsilon\left(\overline{Q_{L}} d_{R}\right)+\text { H.c., }\left(\overline{\ell_{L}} d_{R}\right) \epsilon\left(\overline{Q_{L}} N_{R}\right)+\text { H.c.; } \\
\mathcal{O}_{6}^{L R R L}= & \left(\overline{Q_{L}} u_{R}\right)\left(\overline{N_{R}^{c}} \ell_{L}\right)+\text { H.c. }
\end{aligned}
$$

To this end we have listed only tree generated (TG) operators which conserve baryon number. Note that as $N_{R}$ is a Majorana field, hence $\Gamma^{\mu} \equiv \gamma^{\mu} \gamma^{5}$. All the SM lefthanded quark and lepton $S U(2)_{L}$ doublets are denoted by $Q_{L}, \ell_{L}$ respectively while the right-handed singlet up-type quarks, down-type quarks, and leptons are denoted respectively by $u_{R}, d_{R}, e_{R}$. As per [82] there are 52 dimensionseven independent operators containing sterile or righthanded neutrinos. All these operators carry two units of lepton number, five of which further carry one unit of baryon number, and are thus all non-Hermitian. In the Dirac case, all operators violating lepton number are absent, and we need to replace the Majorana kinetic and mass terms by their Dirac fermion counterparts. Also, in the Dirac case the $\mathcal{O}_{5}$ operator should become $\bar{N} N H^{\dagger} H$.

\section{C. $N_{R}$-DM operators}

The lowest-dimensional operator that can be formed out of the DM and the sterile neutrino fields are dim. 6 fourfermion operators

$$
\mathcal{O}_{\chi N}^{6}=\overline{N_{R}^{c}} \Gamma N_{R}^{\overline{\chi^{c}}} \Gamma \chi,
$$

where $\Gamma \in\left\{1, \gamma^{\mu} \gamma^{5}, \gamma^{5}\right\}$. As one can understand, these operators are suppressed by $1 / \Lambda^{2}$ and hence in the presence of dim.5 DM-SM interaction the production of DM from the heavy neutrinos is negligible and can can safely be ignored. One can certainly write DM-SM interactions following Eq. (11) by replacing the right-handed neutrino field with the SM fermion fields. But as we are considering only DM scalar bilinears, such DM-SM operators shall not arise in a full SM basis [75]. Thus, the sole source of DM production in dim. 6 is via the four-fermion interaction involving heavy neutrinos. With this, we now have all the necessary ingredients to address baryogenesis via (nonthermal) leptogenesis from the decay of the heavy neutrinos together with DM genesis via freeze-in in the early Universe.

\section{NONTHERMAL LEPTOGENESIS}

In the standard vanilla leptogenesis [14] scenario the right-handed neutrinos are produced thermally by scattering processes in the thermal bath or are assumed to be initially in thermal equilibrium. The lower bound on the mass of the lightest right-handed neutrino, in turn, puts a lower bound on the reheat temperature; $M_{1} \gtrsim 10^{10} \mathrm{GeV}$ $[16,85]$. Such a large reheat temperature can lead to gravitino overproduction in supersymmetric scenarios [86]. An elegant alternative to avoid such a catastrophe is to consider nonthermal production of the right-handed neutrinos. In the present case we assume the inflaton $\varphi$ decays exclusively to a pair of RHNs [32,87] via

$$
\mathcal{L} \supset y_{\varphi} \varphi \overline{N_{R}^{c}} N_{R}
$$

where $y_{\phi}$ is the corresponding coupling strength. ${ }^{4}$ The decay width for such a process is

$$
\Gamma_{\varphi} \simeq \frac{y_{\varphi}^{2}}{4 \pi} M_{\varphi} .
$$

After the inflaton condensate has decayed away, the heavy neutrinos dominate the energy density of the Universe. Subsequently, their out-of-equilibrium decay

\footnotetext{
${ }^{4}$ Here we consider the inflaton-Higgs coupling to be absent.
} 
generates the required lepton asymmetry, and reheat the Universe since their decay products, SM lepton and Higgs doublets, thermalize quickly. Thus, the decay of the heavy right-handed neutrinos not only triggers leptogenesis but also produces the thermal bath as well. This leads to interesting consequences contrary to the usual cases where the radiation is produced directly from inflaton decay. Assuming that the energy stored in the inflaton condensate is instantaneously transformed into radiation one finds

$$
T_{\mathrm{RH}}=\left(\frac{90}{8 \pi^{3} g_{\star}}\right)^{1 / 4} \sqrt{\Gamma_{\varphi} M_{\mathrm{pl}}}
$$

which we shall use to parametrize the inflation-heavy neutrino coupling $y_{\varphi}$ in one of our scenarios, where the Hubble parameter at the end of reheating is given by [88]

$$
H\left(T_{\mathrm{RH}}\right)=\left(\frac{8 \pi^{3} g_{\star}}{90}\right)^{1 / 2} \frac{T_{\mathrm{RH}}^{2}}{M_{\mathrm{pl}}} .
$$

It is important to note here that the instantaneous decay of inflaton energy density to radiation energy density is an assumption which may or may not always hold. As a result, we shall treat two cases separately: (i) instantaneous inflaton decay where Eq. (14) holds and the Yukawa coupling $y_{\varphi}$ is parametrized via the reheat temperature $T_{\mathrm{RH}}$, and (ii) noninstantaneous inflaton decay where the Yukawa coupling $y_{\varphi}$ is a free parameter. In the latter case $T_{\mathrm{RH}}$ is defined as the temperature where the inflaton energy density becomes equal to the radiation energy density and can be determined by solving the coupled Boltzmann equations as we shall elaborate [78]. In the later case, however, it is not possible to establish an analytical relation between $y_{\varphi}$ and $T_{\mathrm{RH}}$.

It is possible to define a reheating temperature for the reheating process initiated by the RHNs in the same spirit we define reheating temperature due to inflaton decay [32]

$$
T_{\mathrm{RH}}^{N}=\left(\frac{90}{8 \pi^{3} g^{\star}}\right)^{\frac{1}{4}} \sqrt{\Gamma_{N} M_{\mathrm{pl}}},
$$

where $\Gamma_{N}$ is the decay width in the RHN rest frame. As advocated earlier, we are typically interested in the nonthermal leptogenesis scenario where we assume $T_{\mathrm{RH}}<M_{1}$. For the choice of parameters which gives rise to the right DM abundance with observed baryon asymmetry (together with correct light neutrino mass), we find that $\Gamma_{\varphi} \ll \Gamma_{N}$ always holds as long as $T_{\mathrm{RH}}<M_{1}$. This demonstrates that the time span of the right-handed neutrino dominated Universe is extremely small, and hence the true reheat temperature of the Universe is $T_{\mathrm{RH}}$ due to inflaton decay and not $T_{\mathrm{RH}}^{N}$. It is important to note here, as we are considering $M_{\varphi} \gtrsim 2 M_{1}$, that the RHNs can be considered to be nonrelativistic. Obviously this is an assumption that depends on the inflaton mass. If $M_{\varphi} \gg M_{1}$, the produced RHNs are relativistic and their energy density contributes to the radiation energy density. However that is not the case here because of the chosen mass hierarchy, and the heavy neutrinos are nonrelativistic. Finally, note that once we have $T_{\mathrm{RH}}<M_{i}$ the lepton asymmetry wash-out processes can be neglected as the thermal bath does not have sufficient energy to produce the RHNs. We suppose that the RHN masses are hierarchical, with $M_{1} \ll M_{2}$ similar to vanilla leptogenesis but $M_{1}>T_{\mathrm{RH}}$ such that the lightest heavy neutrino $N_{1}$ with mass $M_{1}$ contributes dominantly to the asymmetry generation. This is precisely due to the fact that any source of lepton number violation at low scale washes out the asymmetries created due to similar violation at high energy scale. Additionally, in our case, we take $M_{\varphi} \gtrsim 2 M_{1}$ and hence inflaton decay into the lightest RHN is more favored kinematically. Thus, if $M_{2}>M_{\varphi} / 2$, then the inflaton only decays exclusively into two $N_{1}$ neutrinos.

\section{A. Light neutrino mass and Casas-Ibarra parametrization}

The extension of the SM particle spectrum with SM singlet RHNs allows us to write its Yukawa interaction with the SM lepton doublet and SM Higgs doublet whereas at the same time, being a neutral SM singlet one cannot forbid its bare mass term which violates the lepton number (an essential ingredient for leptogenesis) by two units. The momentneutral component of the SM Higgs doublet acquires a vacuum expectation value (VEV) leading to the spontaneous breaking of the gauge symmetry; neutrinos which were massless in the SM obtain a Dirac mass which can be written as

$$
m_{D}=\frac{y_{N}}{\sqrt{2}} v
$$

Here, $v=246 \mathrm{GeV}$ denotes the VEV of the SM Higgs field. The presence of this Dirac mass together with the bare mass term of the heavy RHNs can explain the nonzero light neutrino masses through Type-I seesaw $[9,11,89]$. Here, the light-neutrino masses can be expressed as

$$
m_{\nu} \simeq m_{D} M_{N}^{-1}\left(m_{D}\right)^{T},
$$

with $M_{N}$ being the mass of the heavy RHN. The mass eigenvalues and mixing are then obtained by diagonalizing the light-neutrino mass matrix as

$$
m_{\nu}=U^{*}\left(m_{\nu}^{d}\right) U^{\dagger}
$$

with $m_{\nu}^{d}=\operatorname{diag}\left(m_{1}, m_{2}, m_{3}\right)$ consisting of the mass eigenvalues and $U$ being the Pontecorvo-Maki-NakagawaSakata matrix [1] (the charged lepton mass matrix is considered to be diagonal). These discussions suggest the importance of the Yukawa coupling in generating the neutrino masses. The same Yukawa couplings also play a nontrivial role in determining the lepton asymmetry of the 
Universe as they dictate the decay width of RHNs into leptons. In order to obtain a complex structure of the Yukawa coupling which is essential from the leptogenesis point of view we use the well-known Casas-Ibarra (CI) parametrization [90]. Using the CI parametrization one can write the Yukawa coupling as

$$
y_{N}=\frac{\sqrt{2}}{v} U \sqrt{m_{\nu}^{d}} \mathbb{R}^{T} \sqrt{M}
$$

where $\mathbb{R}$ is a complex orthogonal matrix $\mathbb{R}^{T} \mathbb{R}=I$, which we choose as

$$
\mathbb{R}=\left(\begin{array}{ccc}
0 & \cos z & \sin z \\
0 & -\sin z & \cos z
\end{array}\right)
$$

where $z=a+i b$ is a complex angle. In order to obtain $m_{\nu}^{d}=\operatorname{diag}\left(m_{1}, m_{2}, m_{3}\right)$, we consider the lightest neutrino mass eigenvalue to be vanishingly small with $m_{1}=$ $0\left(m_{1}<m_{2}<m_{3}\right)$ for normal hierarchy of the neutrino masses. Then $m_{\nu}^{d}$ is calculable using the best values of solar and atmospheric mass splittings obtained from the latest neutrino oscillation data [1]. Now, the elements of Yukawa coupling matrix, $y_{N}$ for a specific value of $z$ can be obtained for different choices of the heavy neutrino masses. The complex angle $z$ can be chosen in a way that enhances the $C P$ asymmetry while keeping the Yukawa couplings within perturbative limits. The $C P$ asymmetry parameter is defined as

$$
\epsilon_{i}=\frac{\sum_{j} \Gamma\left(N_{i} \rightarrow \ell_{j} H\right)-\sum_{j} \Gamma\left(N_{i} \rightarrow \bar{\ell}_{j} \bar{H}\right)}{\sum_{j} \Gamma\left(N_{i} \rightarrow \ell_{j} H\right)+\sum_{j} \Gamma\left(N_{i} \rightarrow \overline{\ell_{j}} \bar{H}\right)} .
$$

Neglecting flavor effects we can write the final expression for the $C P$-asymmetry as $[14,91]$

$$
\epsilon_{1} \simeq \frac{3}{16 \pi} \frac{\operatorname{Im}\left[\left(y_{N}^{\dagger} y_{N}\right)_{12}^{2}\right]}{\left(y_{N}^{\dagger} y_{N}\right)_{11}}\left(\frac{M_{1}}{M_{2}}\right)
$$

which occurs due to the decay of the lightest right-handed neutrino $N_{1}$. Note that we are working in two RHN setups, hence no summation is involved on the right-hand side of the above equation. Although $N_{2}$ decay can also generate lepton asymmetry, in principle, we consider the asymmetry generated by $N_{2}$ decay or any preexisting asymmetry to be negligible due to strong wash-out effects due to entropy injection. Finally, we would like to give an explicit structure for the complex Yukawa matrix $y_{N}$ for the set of parameters $M_{1}=10^{12} \mathrm{GeV}, M_{2}=10 M_{1}$ with $a=0.3$, $b=0.04$ that provides the desired $C P$ asymmetry satisfying light neutrino mass

$$
y_{N}=\left(\begin{array}{cc}
0.0079+0.0011 i & -0.0109+0.0108 i \\
0.0174+0.0017 i & 0.0517-0.0020 i \\
-0.0009+0.0022 i & 0.0707-0.0004 i
\end{array}\right) .
$$

\section{DARK MATTER PRODUCTION VIA UV FREEZE-IN}

As the SM bath is getting produced from the decay of the lightest right-handed neutrino, the DM can be produced from the thermal bath assuming effective interactions of the form $\bar{\chi} \chi H^{\dagger} H$ in $\operatorname{dim} .5$. This essentially indicates that the DM is getting produced out of equilibrium via freeze-in from the thermal bath through a dim.5 interaction, while at dim.6 it can also be produced directly from RHNs following the interaction in Eq. (11). Since in the presence of dim.5 operators, higher-dimensional interactions (of dimension $d>5$ ) are naturally suppressed; hence we will show the effect of dim.5 and dim.6 interactions on DM production individually. Now, the key requirement for freeze-in $[44,45]$ DM production is to assume that DM was not present in the early Universe. The DM is then produced via annihilation and/or decay of the particles in the thermal bath. ${ }^{5}$ Due to the extremely feeble coupling of the DM with the visible sector particles the DM never really enters into thermal equilibrium. Such feeble interaction is ensured due to higherdimensional operators suppressed by a large cut off scale, as mentioned earlier. Now, the Boltzmann equation (BEQ) for DM production via annihilation or $2 \rightarrow 2$ processes of the form $a, b \rightarrow 1,2$ is then given by [92]

$$
\begin{aligned}
\dot{n_{1}}+3 \mathcal{H} n_{1}= & \int d \Pi_{1} d \Pi_{2} d \Pi_{a} d \Pi_{b}(2 \pi)^{4} \delta^{4} \\
& \left(p_{1}+p_{2}-p_{a}-p_{b}\right)|\mathcal{M}|_{a, b \rightarrow 1,2}^{2} f_{a} f_{b},
\end{aligned}
$$

where $d \Pi_{j}=\frac{d^{3} p_{j}}{2 E_{j}(2 \pi)^{3}}$ are Lorentz invariant phase-space elements, and $f_{i}$ is the phase-space density of particle $i$ with corresponding number density being

$$
n_{i}=\frac{g_{i}}{(2 \pi)^{3}} \int d^{3} p f_{i},
$$

with $g_{i}$ is the internal degrees of freedom (d.o.f.). Here we have made two crucial assumptions: the initial abundance for particle 1 is negligible such that $f_{1} \approx 0$ initially and we also neglect Pauli-blocking/stimulated emission effects, i.e., approximating $1 \pm f_{i} \approx 1$. The BEQ in Eq. (25) can be rewritten as an integral with respect to the centre of mass energy as $[44,68]$

$$
\begin{aligned}
\dot{n}_{1}+3 \mathcal{H} n_{1} & \approx \frac{T}{512 \pi^{6}} \int_{s=4 m_{\chi}^{2}}^{\infty} d s d \Omega P_{a b} P_{12} \\
& \times|\mathcal{M}|_{a b \rightarrow 12}^{2} \frac{1}{\sqrt{s}} K_{1}\left(\frac{\sqrt{s}}{T}\right),
\end{aligned}
$$

\footnotetext{
${ }^{5}$ As we discuss in detail, for dim.6 interactions, DM is produced from RHNs which are not part of the thermal bath but is present in the Universe due to inflaton fields decaying into them.
} 
where $P_{i j}=\frac{1}{2 \sqrt{s}} \sqrt{s-\left(m_{i}+m_{j}\right)^{2}} \sqrt{s-\left(m_{i}-m_{j}\right)^{2}} \rightarrow \frac{\sqrt{s}}{2}$ in the limit $m_{i, j} \rightarrow 0$. The BEQ in terms of the yield or comoving number density $Y_{\chi}=n_{1} / s$ ( $s$ being the entropy density) can be written in the differential form as

$$
\begin{aligned}
-s(T) \cdot \mathcal{H}(T) \cdot T \cdot \frac{d Y_{\chi}^{\mathrm{ann}}}{d T}= & \frac{T}{512 \pi^{6}} \int_{s=4 m_{\chi}^{2}}^{\infty} d s d \Omega P_{a b} P_{12} \\
& \times|\mathcal{M}|_{a b \rightarrow 12}^{2} \frac{1}{\sqrt{s}} K_{1}\left(\frac{\sqrt{s}}{T}\right) .
\end{aligned}
$$

The total yield then turns out to be

$$
\begin{aligned}
Y_{\chi}(T)= & \frac{1}{512 \pi^{6}} \int_{T}^{T_{\max }} \frac{d T^{\prime}}{s\left(T^{\prime}\right) \cdot \mathcal{H}\left(T^{\prime}\right)} \int_{s=4 m_{\chi}^{2}}^{\infty} d s d \Omega\left(\frac{\sqrt{s}}{2}\right)^{2} \\
& \times|\mathcal{M}|_{a b \rightarrow 12}^{2} \frac{1}{\sqrt{s}} K_{1}\left(\frac{\sqrt{s}}{T^{\prime}}\right) .
\end{aligned}
$$

Since we are dealing with pure UV freeze-in case where the DM production is sensitive to high temperature, hence we will ignore masses of all the SM states (as well as the DM) associated with the processes contributing to DM abundance. This is legitimate as long as the reheat temperature and the cut off scale is large compared to the masses involved. In Eq. (29), we consider the maximum temperature of the Universe $T_{\max }$ to be the reheat temperature $T_{\mathrm{RH}}$. Now, the reheat temperature is very loosely bounded, as mentioned before. Typically, the lower bound on $T_{\mathrm{RH}}$ comes from the measurement of light element abundance during $\mathrm{BBN}$, which requires $T_{\mathrm{RH}} \gtrsim$ $1.8 \mathrm{MeV}$ [93]. The upper bound, on the other hand, may emerge from (i) the cosmological gravitino problem $[94,95]$ in the context of the supersymmetric framework that demands $T_{\mathrm{RH}} \lesssim 10^{10} \mathrm{GeV}$ to prohibit thermal gravitino overproduction, and (ii) simple inflationary scenarios that require at most $T_{\mathrm{RH}} \sim 10^{16} \mathrm{GeV}[96,97]$ for a successful inflation. In view of this, the reheat temperature of the Universe can thus be regarded as a free parameter.

\section{THE COUPLED BOLTZMANN EQUATIONS}

Let us recapitulate our scenario so far. For successful leptogenesis, we rely upon the out-of-equilibrium decay of the heavy neutrino $N_{1}$ that is being produced from the decay of inflaton. The out-of-equilibrium decay eventually produces the observed baryon asymmetry via nonthermal leptogenesis. Since inflaton does not decay into radiation directly, right-handed neutrino decay into the SM particles is also responsible for reheating the Universe. The DM, on the other hand, is produced from the SM bath via dim.5 DM-SM interaction through the standard UV freeze-in mechanism. In dim.6 the DM can be produced both from the SM bath and from the right-handed neutrinos

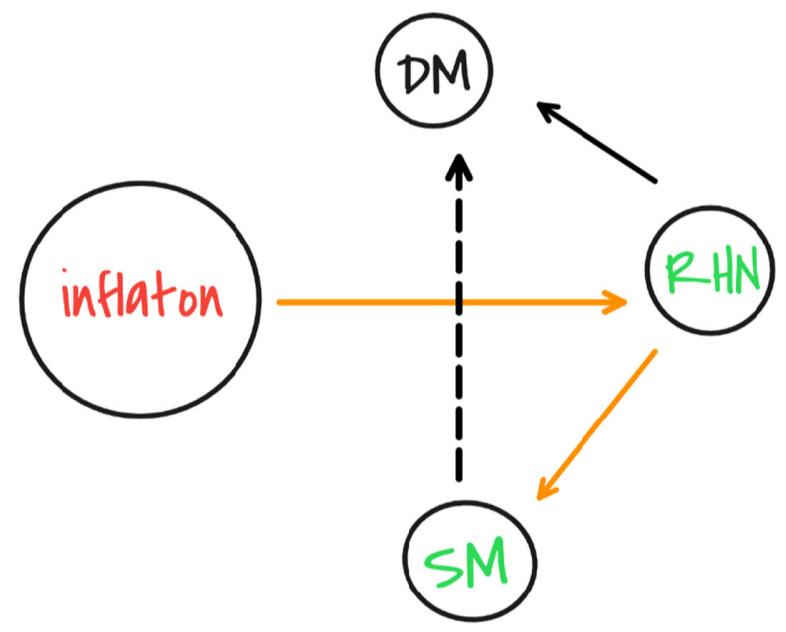

FIG. 1. A schematic view of the framework for production of different components. In dim. 5 there is no interaction between the heavy neutrinos and the DM, while DM-SM interaction is always present for dimensions $d>4$.

via four-fermion interaction. Similar to the previous work [75], we consider only scalar bilinears for DM and hence DM-SM operators do not appear at dim.6 level. Figure 1 shows a cartoon depicting the sources of different components of the Universe in our scenario based on which the coupled BEQ needs to be formulated. Note that, since there is no direct inflaton-DM coupling, the DM can not be produced directly from the inflaton decay. A loop-induced radiative decay of inflaton to a pair of DM is possible in dim. 6 due to the presence of $N N \chi \chi$ operator. Such a process will be suppressed by a factor of $\sim y_{\varphi}^{2} / 16 \pi^{2} \Lambda^{4}$ and can be neglected as $\Lambda \sim \mathcal{O}\left(10^{14}\right) \mathrm{GeV}$ and $y_{\varphi} \sim \mathcal{O}\left(10^{-7}\right)$, required to satisfy relic criteria of $\mathrm{DM}$ in $\mathrm{TeV}$ mass range or below. At dim.5 level no such radiative inflaton decay can arise since we are considering the Higgs-inflaton coupling to be absent. ${ }^{6}$ With this, the set of coupled BEQ reads

$$
\begin{aligned}
\dot{\rho_{\varphi}} & =-3 \mathcal{H} \rho_{\varphi}-\Gamma_{\varphi} \rho_{\varphi} \\
\dot{\rho_{N}} & =-3 \mathcal{H} \rho_{N}+\Gamma_{\varphi} \rho_{\varphi}-\Gamma_{N} \rho_{N} \\
\dot{n}_{B-L} & =-3 \mathcal{H} n_{B-L}+\epsilon \Gamma_{N} n_{N}-\Gamma_{\mathrm{ID}} n_{B-L} \\
\dot{\rho}_{R} & =-4 H \rho_{R}+\Gamma_{N} \rho_{N} \\
\dot{n}_{\chi} & =-3 \mathcal{H} n_{\chi}+\langle\sigma v\rangle n_{\mathrm{eq}}^{2},
\end{aligned}
$$

where $\epsilon$ is the $C P$-asymmetry generated by the lightest RHN decay [as in Eq. (23)] and $\Gamma_{\mathrm{ID}}$ is the inverse decay rate, and the DM number density evolution follows from Sec. IV. Going forward we are going to ignore the inverse decay rate. Since the RHNs are assumed to be nonrelativistic hence $n_{N}=\rho_{N} / m_{N}$ where in our case

${ }^{6} \mathrm{DM}$ production via $2 \rightarrow 2$ annihilation of the inflaton mediated via gravitons are studied in [98-101]. 
$m_{N} \equiv M_{1}$. Note that in the BEQ governing the DM abundance we have the equilibrium number density $n_{\mathrm{eq}}^{2}$ which is applicable for dim.5 as the DM is produced only from the thermal bath. In case of dim.6 (where we ignore the dim.5 operators), as we will see later, one has to replace the equilibrium number density with the heavy neutrino number density as the RHNs become the source of DM production via Eq. (11). Next we are going to make appropriate variable transformations in order to solve the set of coupled equations.

\section{A. Variable transformation and initial conditions}

It is convenient to make suitable variable transformations while solving Eq. (30). We make the following set of transformations by scaling the energy and number densities with the scale factor $a[22,32]$

$$
\begin{aligned}
E_{\varphi} & =\rho_{\varphi} a^{3}, \\
E_{N} & =\rho_{N} a^{3}, \\
\tilde{N}_{B-L} & =n_{B-L} a^{3}, \\
R & =\rho_{R} a^{4}, \\
X & =n_{\chi} a^{3} .
\end{aligned}
$$

We also define

$$
y=\frac{a}{a_{I}}
$$

as the ratio of scale factors and assume $a_{I}=1$. The factor $a_{I}$ is chosen as the initial value of the scale factor while $y$ is equivalent to the time variable. With this the Hubble parameter reads

$$
\mathcal{H}=\sqrt{\frac{8 \pi\left(E_{\varphi} a_{I} y+E_{N} a_{I} y+R\right)}{3 M_{\mathrm{pl}}^{2} a_{I}^{4} y^{4}}} .
$$

Instead of the temperature $T$ we use a dimensionless quantity $z=M_{1} / T$ as a variable and define it as a function of $R$ mentioned in Eq. (20)

$$
z=\frac{M_{1}}{T}=\frac{M_{1}}{\left(30 \rho_{R} / \pi^{2} g_{\star}\right)^{\frac{1}{4}}}=M_{1}\left(\frac{\pi^{2} g_{\star}}{30 R}\right)^{\frac{1}{4}} y a_{I} .
$$

In terms of theses rescaled variables Eq. (30) can be written as

$$
\begin{aligned}
E_{\varphi}^{\prime} & =-\frac{\Gamma_{\varphi}}{\mathcal{H}} \frac{E_{\varphi}}{y}, \\
E_{N}^{\prime} & =\frac{\Gamma_{\varphi}}{\mathcal{H}} \frac{E_{\varphi}}{y}-\frac{\Gamma_{N}}{\mathcal{H} y} E_{N}, \\
\tilde{N}_{B-L}^{\prime} & =\frac{\Gamma_{N}}{\mathcal{H} y} \epsilon \frac{E_{N}}{M_{1}}, \\
R^{\prime} & =\frac{\Gamma_{N} a_{I}}{\mathcal{H}} E_{N}, \\
X^{\prime} & =\frac{1}{16 \pi^{3} \Lambda^{2}} \frac{y^{2}}{\mathcal{H}}\left(\frac{M_{1}}{z}\right)^{6} .
\end{aligned}
$$

In the above set of equations all the primes correspond to the derivative with respect to $y=a / a_{I}$. In obtaining the initial energy density for the inflaton we use the condition

$$
\Gamma_{\varphi}=\mathcal{H}\left(a_{I}\right)
$$

that leads to

$$
E_{\varphi_{I}}=\frac{3}{8 \pi} M_{\varphi}^{2} M_{\mathrm{pl}}^{2}
$$

Except for the inflaton, which has an initial energy density, all other quantities are being produced from different sources. Hence we choose the initial values of the variables appearing in Eq. (34) as

$$
\begin{gathered}
\tilde{N}_{(B-L)_{I}}=0 ; \quad E_{\varphi}=E_{\varphi_{I}} ; \\
R_{I}=0 ; \quad E_{N_{I}}=0 ; \quad X_{I}=0 .
\end{gathered}
$$

The observed baryon asymmetry depends on the final $B-L$ abundance which can be derived from the redefined $\tilde{N}_{B-L}$ via

$N_{B-L}=\frac{n_{B-L}}{n_{\gamma}}=\left[\frac{\pi^{4}}{60 \zeta(3)}\right]\left(\frac{30}{\pi^{2} g_{\star \rho}}\right)^{1 / 4} g_{\star \rho} \tilde{N}_{B-L} R^{-3 / 4}$,

where we have recasted $n_{\gamma}=g_{\star} \zeta(3) T^{3} / \pi^{2}$ in terms of $\rho_{\gamma}=$ $\left(\pi^{2} / 30\right) g_{\star} T^{4}$ and also assumed

$$
T=\left(\frac{30 \rho_{R}}{\pi^{2} g_{\star}}\right)^{1 / 4}
$$

which is allowed since the heavy neutrinos are nonrelativistic. ${ }^{7}$ The predicted baryon to photon number ratio has to be compared with the value $\eta_{B}$ measured at the recombination epoch. It is related to $N_{B-L}$ by $[14,32]$

\footnotetext{
${ }^{7}$ If the produced heavy neutrinos were relativistic then $T=\left(\frac{30\left(\rho_{R}+\rho_{N}\right)}{\pi^{2} g_{\star}}\right)^{1 / 4}$.
} 


$$
\eta_{B}=\left(\frac{a_{\mathrm{sph}}}{f}\right) N_{B-L}
$$

where $a_{\mathrm{sph}}=28 / 79$ is the fraction of $B-L$ asymmetry converted into a baryon asymmetry by sphaleron processes and $f=N_{\gamma}^{\mathrm{rec}} / N_{\gamma}^{\star}=2387 / 86$ is the dilution factor calculated assuming standard photon production from the onset of leptogenesis until recombination [14]. The DM abundance can be calculated via

$$
\begin{aligned}
\Omega_{\chi} h^{2}= & \left(\frac{1.53 \times 10^{8}}{g_{\star s}}\right)\left(\frac{30}{\pi^{2} g_{\star \rho}}\right)^{1 / 4} \\
& \times\left(\frac{g_{\star \rho} \pi^{4}}{60 \zeta(3)}\right) g_{\star s} m_{\chi} X\left(y_{\infty}\right) R\left(y_{\infty}\right)^{-3 / 4},
\end{aligned}
$$

where we have used $s \approx 1.8 g_{\star s} n_{\gamma}$ [42] to calculate the comoving entropy density and $y_{\infty}$ corresponds to the asymptotic value of $y=a / a_{I}$.

\section{RESULTS AND DISCUSSIONS}

In this section we would like to discuss and summarize our findings. We mainly focus on dim.5 DM-SM interaction where the DM is produced via UV freeze-in from interaction of the form in Eq. (8). Later we shall briefly discuss the effect of dim.6 $N_{R}$-DM operators on DM yield. As clarified in the beginning, we investigate the instantaneous and noninstantaneous inflaton decay cases separately and point out the differences thereof. We begin with the instantaneous inflaton-decay scenario and illustrate how the resulting parameter space can give rise to the observed DM relic abundance, together with the correct baryon asymmetry. Then we will move on to the more general noninstantaneous decay case and make a comparative analysis between the two.

\section{A. Instantaneous inflaton decay}

In the instantaneous inflaton decay approximation Eq. (14) holds and hence the inflaton-heavy neutrino coupling is automatically determined once $T_{\mathrm{RH}}$ is chosen. Keeping this in mind, let us first look into the dependence of the yield of $\left\{E_{N}, E_{\varphi}, R\right\}$ on the heavy neutrino mass $M_{1}$ and reheat temperature $T_{\mathrm{RH}}$, as these two parameters mainly control the above three quantities while the effective cut off scale becomes important for the DM relic abundance. As mentioned before, we are interested in pure UV freeze-in production of the DM. This entails to only four-point contact interaction for the DM production via $H H \rightarrow \chi \chi$ process where $H$ is the SM-like Higgs doublet. However, one has to take into account of the fact that before electroweak symmetry breaking we have

$$
H=\left(\begin{array}{c}
\phi^{+} \\
\phi^{0}
\end{array}\right)
$$

where the massless Goldstone bosons (GB) $\phi^{ \pm, 0}$ are the propagating d.o.f.s. One can also note that in $\operatorname{dim} .5$ we have $N N \rightarrow H H$ process via the operator in Eq. (8) which also contributes to the thermal bath. But the out-of-equilibrium $N \rightarrow \ell H$ decay will always dominate over pair annihilation of $N$ with the latter being $\Lambda$ suppressed. Hence, heavy neutrino decay is considered to be the dominant source of entropy production.

The variation of $\left\{E_{N}, E_{\varphi}, R\right\}$ as function of $y=a / a_{I}$ is demonstrated in Fig. 2. We normalize all the yields with the initial inflaton energy such that it turns out to be a dimensionless quantity. In all these plots we have kept the cut off scale $\Lambda$ and inflaton mass $M_{\varphi}$ fixed at values $10^{16} \mathrm{GeV}$ and $10^{13} \mathrm{GeV}$ respectively. These choices are purely ad hoc but ensuring the fact that $\varphi \rightarrow N_{1} N_{1}$ decay channel is always open. Later we shall discuss the possible choices of the parameters which are consistent with the observed baryon asymmetry and DM abundance. In all these plots we ensure that the measured light neutrino mass is obtained (by employing Casas-Ibarra parametrization). At dim.5 the thermally averaged $2 \rightarrow 2$ cross section for $\mathrm{DM}$ production is given by

$$
\langle\sigma v\rangle \simeq \frac{1}{\Lambda^{2}}
$$

considering a pure UV freeze-in scenario where we ignore masses of the DM and the SM fields with respect to the cutoff scale and the reheat temperature. ${ }^{8}$ With this assumptions incorporated here we see the following features:

(a) In the top left panel of Fig. 2 we show the variation of $E_{N}$ with $y=a / a_{I}$ for four different choices of the heavy neutrino mass $M_{1}=\left\{10^{9}, 10^{10}, 10^{11}, 10^{12}\right\} \mathrm{GeV}$ in red, green, blue, and black respectively for a fixed reheat temperature of $10^{8} \mathrm{GeV}$. Here we see for a larger $M_{1}$ the plateau region is wider. This is because for heavier RHN, corresponding Dirac-Yukawa coupling is comparatively large, hence its decay starts competing with the production from a much earlier epoch leading to a wider plateau region. Note that all these curves start falling sharply around the same $y=$ $a / a_{I}$ because of the fixed reheat temperature that keeps the inflaton-heavy neutrino decay unchanged as $\Gamma_{\varphi} \propto T_{\mathrm{RH}}^{2}$. As a consequence, when the inflaton decay is completed, the heavy neutrino production stops and $E_{N}$ falls. For a low mass $N_{1}$ the yield is larger simply because a smaller $M_{1}$ gives rise to a comparatively smaller decay width for the RHN. As a result, the

\footnotetext{
${ }^{8}$ In a more general way one can define [74,102] the thermally averaged cross-sections as: $\langle\sigma v\rangle=\frac{T^{n}}{\Lambda^{n+2}}$ with the operator mass dimension $d=5+n / 2$ for $n \geq 0$ and even.
} 

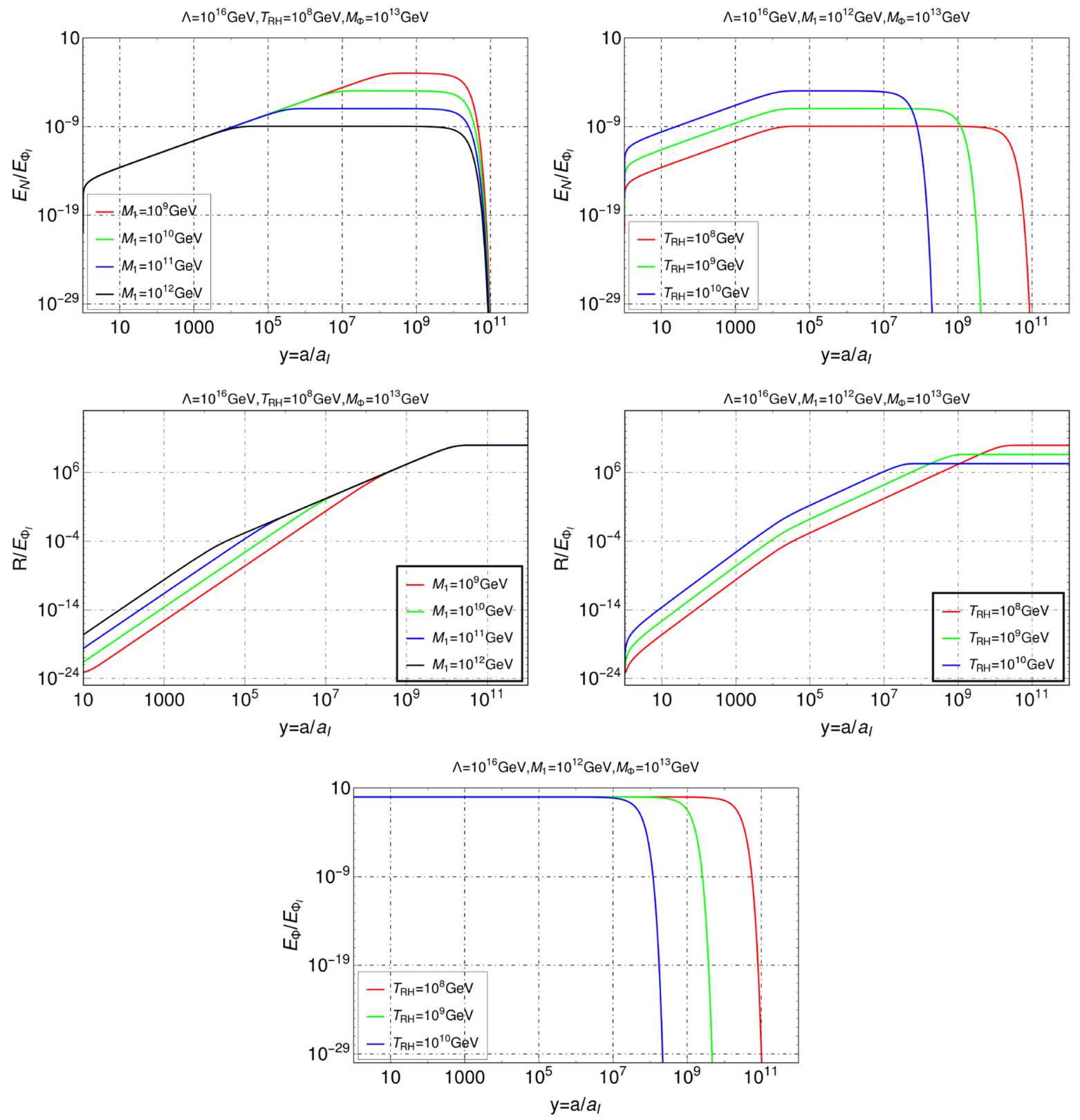

FIG. 2. Top left: Variation of $E_{N}$ with the ratio of scale factors $y=a / a_{I}$ for different choices of the heavy neutrino mass $M_{1}$ for a fixed reheat temperature $T_{\mathrm{RH}}=10^{8} \mathrm{GeV}$. Top right: Same as left but for a fixed $M_{1}=10^{12} \mathrm{GeV}$ with different reheat temperature. Middle left: Radiation $R$ as a function of $y=a / a_{I}$ for different choices of $M_{1}$ at a fixed reheat temperature $T_{\mathrm{RH}}=10^{8} \mathrm{GeV}$. Middle right: Same as middle left but for a fixed $M_{1}=10^{12} \mathrm{GeV}$ with different choices of $T_{\mathrm{RH}}$. Bottom: Variation of $E_{\varphi}$ with $y=a / a_{I}$ for a fixed $M_{1}$ with different choices of the reheat temperature. In all these plots the effective scale is considered to be $\Lambda=10^{16} \mathrm{GeV}$ and an inflaton mass of $M_{\Phi}=10^{13} \mathrm{GeV}$ for illustration.

decay rate starts competing with the production at a much later epoch leading to a larger $N_{1}$ yield (and a smaller plateau).

(b) The effect of reheat temperature on the heavy neutrino yield is shown in the top right panel of Fig. 2. For a fixed $M_{1}$ the heavy neutrino decay width $\Gamma_{N}$ is fixed. Now, a larger $T_{\mathrm{RH}}$ results in larger $\Gamma_{\varphi}$. This implies that the inflaton decay $\varphi \rightarrow N N$ completes earlier for a large $T_{\mathrm{RH}}$. Thus, for large reheat temperature we see the curves start falling at a lower $y$ showing a smaller plateau since the production of RHN becomes comparable with its decay over a shorter period of time. For large reheat temperature, the inflaton decay width is larger and hence its decay into RHNs gets completed at an earlier epoch. For smaller $T_{\mathrm{RH}}$, on the other hand, the plateau is also wider as the inflaton 
decay takes place over a longer epoch. Therefore, in this case, the RHN decay can remain comparable to its production over a longer epoch. For large $T_{\mathrm{RH}}$ the heavy neutrino yield is also larger as $E_{N} \propto \Gamma_{\varphi}$.

(c) In the middle left panel of Fig. 2 we show the behavior of radiation with $y$ for a fixed reheat temperature. Here we see for larger $M_{1}$ the radiation yield is large as $R \propto \Gamma_{N} \propto M_{1}$. However, as the reheat temperature is fixed and so is the inflaton decay width, the heavy neutrino production stops as soon as the inflaton decay is completed. This results in saturation of the radiation yield at $y \gtrsim 10^{9}$. Also, as the entire energy contained in the inflaton field (whose mass is fixed) gets transferred into radiation eventually, all the curves corresponding to different values of $M_{1}$ merge for higher values of $y$. For a particular $M_{1}=10^{12} \mathrm{GeV}$ we see $R$ increases initially up to $y \sim 10^{4}$ during which the rate of production of the RHN dominates over its decay. Then the radiation yield rises linearly (though at a rate slower than initially) as RHN starts to decay until $y \sim 10^{10}$ where the decay rate of the RHN starts overcoming its rate of production. Finally, at $y \gtrsim$ $10^{10}$ the yield saturates when practically the RHN production has stopped as the inflaton decay is over and the RHN decay into the radiation is also completed. It is possible to make an one to one correspondence with the top left panel for the same choice of $M_{1}$. In the middle right panel we show the effect of varying reheat temperature on the radiation yield. As for large $T_{\mathrm{RH}}$ the inflaton decays faster to the RHN. This results in an earlier drop in the heavy neutrino yield. As a result, for a fixed $M_{1}$, the radiation yield from the RHN decay is also smaller. The fact that the heavy neutrino yield drops earlier for larger $T_{\mathrm{RH}}$, is exactly reflected in the blue curve where we see the radiation starts saturating earlier than the other two. Hence the asymptotic yield of radiation for small $T_{\mathrm{RH}}$ (and hence smaller $\Gamma_{\varphi}$ ) wins over those due to comparatively larger $T_{\mathrm{RH}}$ (and hence larger $\Gamma_{\varphi}$ ).

(d) Finally in the bottom panel of Fig. 2 we show the evolution of the inflaton yield for different choices of the reheat temperature at a fixed inflaton mass. Since $\Gamma_{\varphi} \propto T_{\mathrm{RH}}^{2}$ hence a larger choice of the reheat temperature causes a quick decay of the inflaton to a pair of $N_{1}$ as shown by the blue curve. The consequence of this on the heavy neutrino yield (and hence on the radiation yield) are already shown in the top and middle panels. As expected, changing $M_{1}$, keeping $M_{1}<m_{\varphi} / 2$, without changing $T_{\mathrm{RH}}$ does not affect the inflaton yield.

The evolution of the different components with $y=a / a_{I}$ are shown together in Fig. 3. Here we see trends similar to what we already have observed in Fig. 2. The asymptotic value of the yields corresponding to $\tilde{N}_{B-L}$ and $X$ give rise to

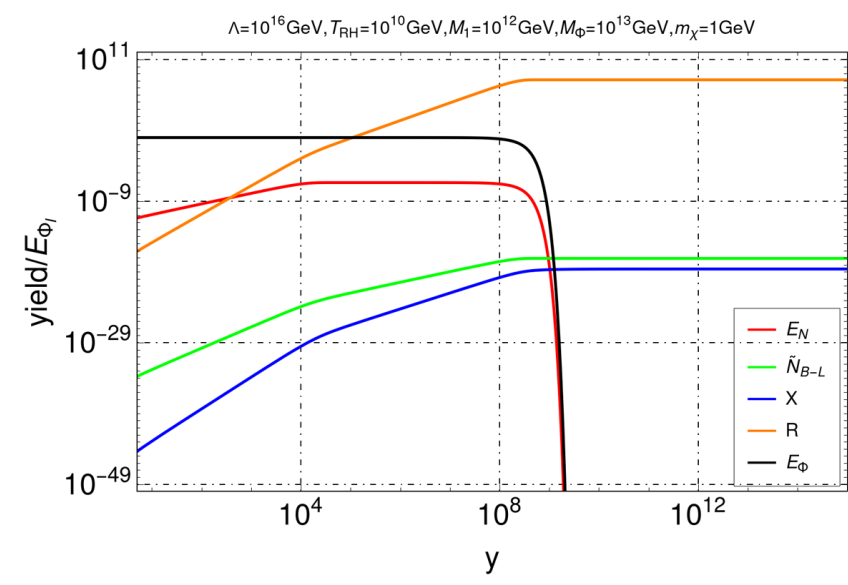

FIG. 3. The evolution of $E_{N}, E_{\varphi}, R, \tilde{N}_{B-L}$ and $X$ are shown for $\left\{\Lambda, M_{1}, T_{\mathrm{RH}}\right\}=\left\{10^{16}, 10^{10}, 10^{9}\right\} \mathrm{GeV}$ for a fixed inflaton mass $M_{\varphi}=10^{13} \mathrm{GeV}$ and DM mass of $m_{\chi}=1 \mathrm{GeV}$. The DM abundance and baryon asymmetry are satisfied at the asymptotic value of the corresponding yield for $X$ and $N_{B-L}$ respectively.

correct baryon asymmetry and DM abundance (for a DM mass of $1 \mathrm{GeV}$ ) at present epoch. For a large reheat temperature one has to choose a larger $\Lambda$ in order to satisfy the DM relic abundance for a fixed DM mass as for pure $\mathrm{UV}$ freeze-in the DM yield goes as $\sim M_{\mathrm{pl}} T_{\mathrm{RH}} / \Lambda^{2}$ for the case of dim.5 DM-SM interactions. For DM production via dim.5 DM-SM interaction it has already been shown that $\Lambda \simeq 10^{16} \mathrm{GeV}$ can satisfy the right relic density for $T_{\mathrm{RH}} \gtrsim$ $10^{9} \mathrm{GeV}$ [75]. As the DM yield is built solely from the thermal bath (radiation) at dim.5 level hence the orange and blue curves, corresponding to radiation and DM yield respectively show the same characteristic evolution. However, as radiation starts to get produced earlier from $N_{1}$ decay, hence the initial yield for radiation is larger compared to that of the DM. As $\tilde{N}_{B-L}$ is generated from the heavy neutrino decays, the final asymmetry becomes saturated exactly at the point when all the $N_{1}$ decay is completed. This is visible by comparing the black and green curves. Inflaton decay being the only source of heavy neutrino production the red and black curves fall at the same point. Since RHN has sizeable Yukawa coupling for such high scale Type-I seesaw realization, they can decay quickly leading to quick depletion in their number density once their production from inflaton decay stops. One can also notice that all the curves except for the black one (which corresponds to the inflaton) has a 'knee' around $y \sim 10^{4}$. This occurs due to the entropy injection from the decay of the heavy neutrino when the RHN decay starts becoming comparable to its production. In all these plots the The effect of maximum temperature during reheating has been taken into account as the maximum temperature $T_{\max }$ corresponds to $y_{\max } \simeq 1.4$ (see Appendix). Production of the right amount of $N_{B-L}$ will lead to the observed 

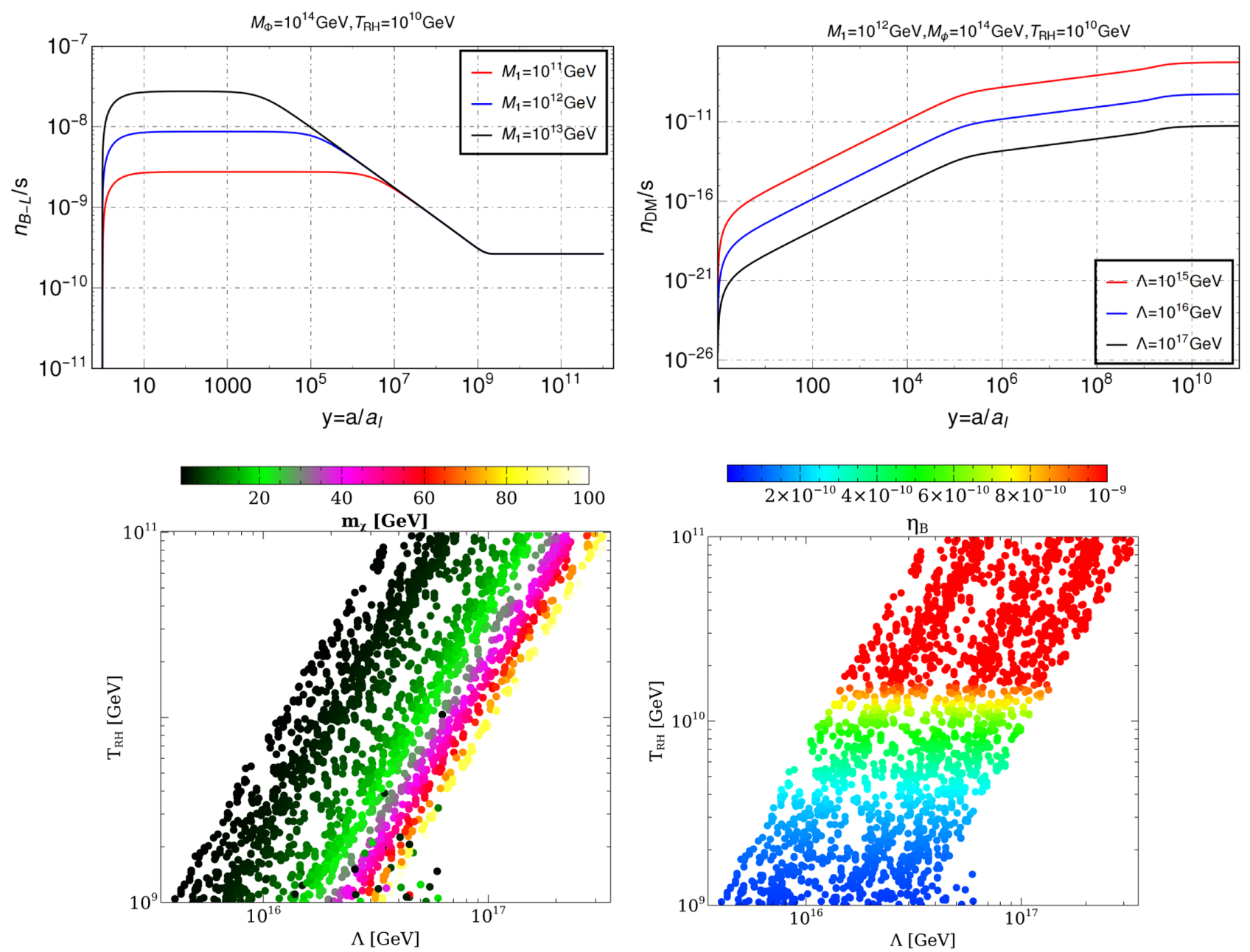

FIG. 4. Top Left: Evolution of $n_{B-L} / s$ with $y=a / a_{I}$ is shown for different choices of the heavy nautrino mass $M_{1}:\left\{10^{11}, 10^{12}, 10^{13}\right\} \mathrm{GeV}$ in black, red and blue respectively for a fixed reheat temperature $T_{\mathrm{RH}}=10^{10} \mathrm{GeV}$ and inflaton mass $M_{\varphi}=10^{14} \mathrm{GeV}$. The thick black dashed line shows the $N_{B-L}$ required to produce observed baryon asymmetry via Eq. (39). Top Right: For the same set of parameters as in the left panel the evolution of DM number density is shown for $\Lambda:\left\{10^{15}, 10^{16}, 10^{17}\right\} \mathrm{GeV}$ in red, blue and black respectively. Bottom Left: Relic density allowed parameter space in $T_{\mathrm{RH}}-\Lambda$ bidimensional plane for $M_{1}=10^{12} \mathrm{GeV}$ and $M_{\varphi}=10^{13} \mathrm{GeV}$ where different DM masses are shown in different colors. Bottom Right: Relic density allowed parameter space in $T_{\mathrm{RH}}-\Lambda$ bidimensional plane for $M_{1}=10^{12} \mathrm{GeV}$ and $M_{\varphi}=10^{13} \mathrm{GeV}$ where different colors correspond to the baryon asymmetry $\eta_{B}$.

baryon asymmetry $\eta_{B}$ following Eq. (39). The evolution of the $B-L$ asymmetry for dim.5 interaction is shown in the top left panel of Fig. 4. Here we have depicted how $n_{B-L} / s$ evolves with the dimensionless quantity $y=a / a_{I}$ for three different masses of the heavy neutrino $N_{1}$. We notice very interesting dynamics of the yield in this case. As $\Gamma_{N} \gg \Gamma_{\varphi}$, hence the heavy neutrinos immediately start decaying after they get produced from the inflaton. As a result, $n_{B-L} / s$ builds up with $y$ at the very beginning $y \sim 1$. The $B-L$ yield remains fixed up to $y \sim 10^{6}$ (for the blue curve) since in this period the radiation yield is small as one can confirm from the middle left panel plot of Fig. 2. Beyond $y \sim 10^{6}$, as the RHN decay becomes comparable to its production, the radiation builds up faster due to entropy injection from the RHN decay. This results in the rapid fall of $n_{B-L} / s$. At $y \sim 10^{9}$ the RHN decay is completed resulting in a constant $B-L$ whence no more entropy injection is possible. In the top right panel of Fig. 4 we show how the DM yield $n_{\mathrm{DM}} / s$ evolves with $y=a / a_{I}$ for the same set of parameters as in the top left panel. As the DM number density is a function of $\propto 1 / \Lambda^{2}$, hence a larger $\Lambda$ results in a suppressed DM yield. For a fixed DM mass, this implies, a larger $\Lambda$ calls for a smaller $T_{\mathrm{RH}}$ in order to satisfy the observed relic abundance since in dim.5 the DM abundance for pure UV freeze-in can approximately be given by $\Omega_{\chi} h^{2} \sim m_{\chi} T_{\mathrm{RH}} / \Lambda^{2}$. One can also notice the bulge at $y \sim 10^{5}$ which we also observed in the DM yield $X$ in Fig. 3 due to entropy injection in the thermal bath from heavy neutrino decay. The DM and the $n_{B-L}$ yield shows an exact complementary behavior as the DM is always being 
produced from the thermal bath and finally becomes constant while $n_{B-L}$ is produced from the decay of the RHN which then saturates at $y \rightarrow \infty$ once the decay is completed. To produce the right relic abundance for the DM via UV freeze-in at dim.5 level, together with a successful nonthermal leptogenesis, one has to choose the free parameters accordingly. In the bottom panel of Fig. 4 we show the parameter space that satisfies correct relic abundance for different DM masses. Here we fix the inflaton mass $M_{\varphi}=10^{13} \mathrm{GeV}$ and heavy RHN mass $M_{1}=10^{11} \mathrm{GeV}$, and vary the rest of the parameters in the following range

$$
\begin{aligned}
& m_{\chi}:\{1-100\} \mathrm{GeV} ; \quad \Lambda:\left\{10^{15}-10^{17}\right\} \mathrm{GeV} ; \\
& T_{\mathrm{RH}}:\left\{10^{9}-10^{11}\right\} \mathrm{GeV} .
\end{aligned}
$$

In the bottom left panel we show the DM relic density allowed region in $T_{\mathrm{RH}}-\Lambda$ plane where the coloring is done over the DM mass. Here we see, for a fixed cut off scale $\Lambda$, a smaller DM mass requires larger $T_{\mathrm{RH}}$ in order to satisfy the observed abundance. This is expected as the relic abundance in dim.5 (as we mentioned earlier) varies as $\Omega_{\chi} h^{2} \sim m_{\chi} T_{\mathrm{RH}} / \Lambda^{2}$. Hence a large DM mass needs a smaller reheat temperature to attain the right relic density. This also implies, for $\mathrm{DM}$ mass $\sim \mathcal{O}(\mathrm{TeV}), T_{\mathrm{RH}}<$ $10^{10} \mathrm{GeV}$ to satisfy the relic abundance in order to keep $\Lambda<M_{\mathrm{pl}}$. It is possible to achieve the observed baryon asymmetry $\eta_{B}$ within this range of $T_{\mathrm{RH}}$ as is evident from the bottom right panel of Fig. 4. Here we show the relic density allowed points in $T_{\mathrm{RH}}-\Lambda$ plane where the coloring is with $\eta_{B}$. We see correct baryon asymmetry is obtained for a very narrow range of $T_{\mathrm{RH}} \sim 10^{10} \mathrm{GeV}$. As $T_{\mathrm{RH}}$ determines the inflaton decay width, hence a large $T_{\mathrm{RH}}$ causes a larger inflaton decay width resulting in larger $N_{1}$ abundance (as one can also notice in the top left panel of Fig. 2). This results in a larger $\eta_{B}$ in turn. Varying $\Lambda$ for a fixed cut off scale does not affect the baryon asymmetry as one should expect. Note that we have chosen DM mass in the $\mathrm{GeV}-\mathrm{TeV}$ window, both in benchmark cases as well as in scans. Since the DM relic is generated via UV freeze-in, it is possible to produce supermassive nonthermal DM within the present framework by carefully tuning $T_{\mathrm{RH}}$ and $\Lambda$ such that with the same choice of parameters one can also satisfy the observed baryon asymmetry. For example, in case of dim.5, it is possible to produce $1 \mathrm{PeV} \mathrm{DM}$ for $\Lambda \sim 10^{17} \mathrm{GeV}$ and $T_{\mathrm{RH}} \sim 10^{7} \mathrm{GeV}$ with $\eta_{B}$ of the correct order. $^{9}$

Before moving on to the next section we would like to comment on the consistency of our rigorous numerical

\footnotetext{
${ }^{9}$ Production of supermassive FIMPs together with light neutrino mass generation and leptogenesis have been explored in the context of UV-complete models [103,104].
}

analysis in determining the final (observed) baryon asymmetry $\eta_{B}$ following Eq. (39), with simple analytical estimates. Under the assumption that right-handed neutrinos decay almost instantaneously after getting produced from inflaton decay, the baryon asymmetry has the wellknown analytical expression [22,24,25,87]

$$
\frac{n_{B}}{s} \simeq-\frac{28}{79} \frac{3}{2} \frac{T_{\mathrm{RH}}}{m_{\varphi}} \cdot \mathcal{B} \cdot \epsilon_{1}
$$

which was derived in the context of inflaton field and leptogenesis without the presence of DM in the same framework. The branching ratio of the inflaton decays into RHNs in Eq. (43) namely, $\mathcal{B}=1$ since the inflaton decays only into the RHNs. In Fig. 5 we have plotted the baryon asymmetry $\eta_{B}$ as a function of the reheating temperature for a fixed RHN and inflaton mass. We find, the asymmetry obtained from the exact numerical solution, (shown by the red curve) is slightly less than the one obtained from the approximate analytical solution (shown by the black curve). To quantify, for $T_{\mathrm{RH}}=10^{9} \mathrm{GeV}$ the analytical estimation gives $\eta_{B}^{\text {analyt }} \approx 1.46 \times 10^{-10}$ while doing the explicit numerical calculation we find $\eta_{B}^{\text {numr }} \approx 5 \times 10^{-12}$. The smallness in baryon asymmetry obtained in exact numerical treatment is expected as entropy release is properly incorporated, which is absent in instantaneous right-handed neutrino decay approximation. While this shows the overall agreement in the behavior of baryon asymmetry obtained from analytical and numerical calculations, it also points out the necessity of performing a full numerical analysis to get a better estimate of baryon asymmetry. While the rise in baryon asymmetry with the reheating temperature for the analytical solution is expected from Eq. (43), a similar rise in the numerical solution can

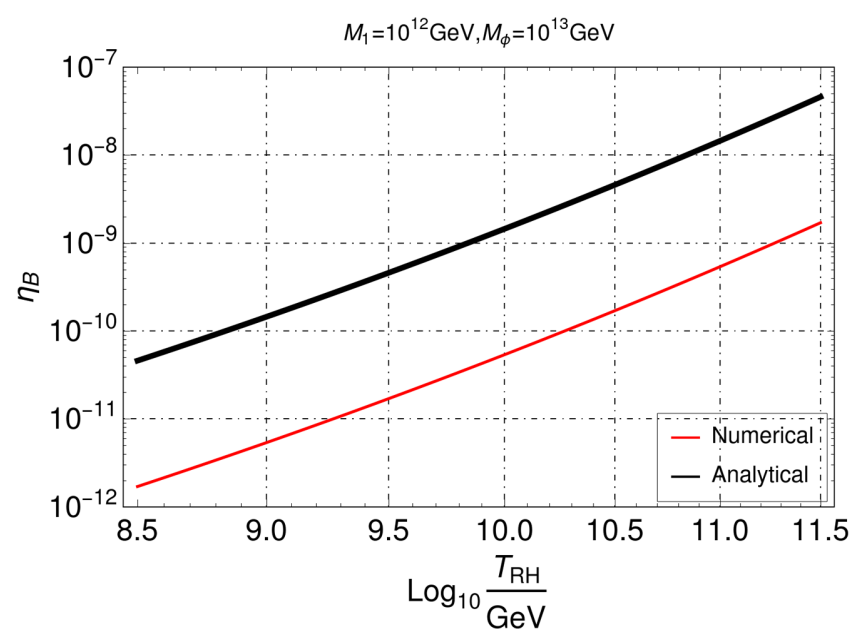

FIG. 5. A comparison of analytically obtained baryon asymmetry (black) with that obtained numerically (red) by solving the coupled BEQ [Eq. (34)] for a fixed heavy neutrino and inflaton mass. 
be explained from the definition of reheat temperature in instantaneous inflaton decay approximation in Eq. (14) and the coupled BEQ in (34). A larger $T_{\mathrm{RH}}$, will result in a larger decay width of the inflaton [as per Eq. (14)], leading to larger abundance of $N_{1}$ (as we can see from the top right panel of Fig. 2). With larger $N_{1}$ abundance we will have a larger lepton asymmetry, which, in turn, shall give rise to an increase in the baryon asymmetry.

\section{Effect of dimension-six operator on DM yield and leptogenesis}

In the presence of dim.5 interactions, the operators which arise at the level of dim. 6 become subdominant. Therefore, in order to single out the effect of dim.6 interactions here we consider dim.5 DM-SM operators to be absent. Since we are considering only scalar DM bilinears, therefore gauge invariant dim.6 DM-SM operators do not arise. This leaves pair annihilation of the heavy neutrinos via $N N \chi \chi$ interaction as the only source for DM. On incorporating this in the yield equation, one ends up with the following set of BEQs

$$
\begin{aligned}
E_{\varphi}^{\prime} & =-\frac{\Gamma_{\varphi}}{\mathcal{H}} \frac{E_{\varphi}}{y} \\
E_{N}^{\prime} & =\frac{\Gamma_{\varphi}}{\mathcal{H}} \frac{E_{\varphi}}{y}-\frac{\Gamma_{N}}{\mathcal{H} y} E_{N} \\
\tilde{N}_{B-L}^{\prime} & =\frac{\Gamma_{N}}{\mathcal{H} y} \epsilon \frac{E_{N}}{M_{1}} \\
R^{\prime} & =\frac{\Gamma_{N} a_{I}}{\mathcal{H}} E_{N} \\
X^{\prime} & =\frac{\langle\sigma v\rangle_{N N \rightarrow \chi \chi}}{\mathcal{H}} \frac{E_{N}^{2}}{M_{1}^{2}} y^{-4},
\end{aligned}
$$

where $\langle\sigma v\rangle_{N N \rightarrow \chi \chi}$ is the pair annihilation cross section of the heavy neutrinos to DM. One should note here the presence of the $E_{N}^{2} / M_{1}^{2}$ term which accounts for the DM production from the heavy neutrino. As the heavy neutrinos are always out-of-equilibrium, we can write

$$
(\sigma v)_{N N \rightarrow \chi \chi} \approx \frac{M_{1}^{2}}{2 \pi \Lambda^{4}} .
$$

On the other hand, several operators connecting the heavy neutrinos with the SM fields appear at dim.6 as mentioned in Sec. II. However, the dominant source of leptogenesis can still be considered to be due to $N_{1} \rightarrow \ell H$ decay as $2 \rightarrow 2$ scattering processes involving $N_{1}$ in the initial or the final states are suppressed for $M_{1}>T_{\mathrm{RH}}$. With this, we can now solve the set of BEQs in Eq. (44). The evolution of yield for dim.6 interactions with $y=a / a_{I}$ is shown in Fig. 6 for a fixed cut off scale, reheat temperature, inflaton mass and lightest right-handed neutrino mass. For a DM mass of $10 \mathrm{GeV}$, the DM yield (shown by the blue

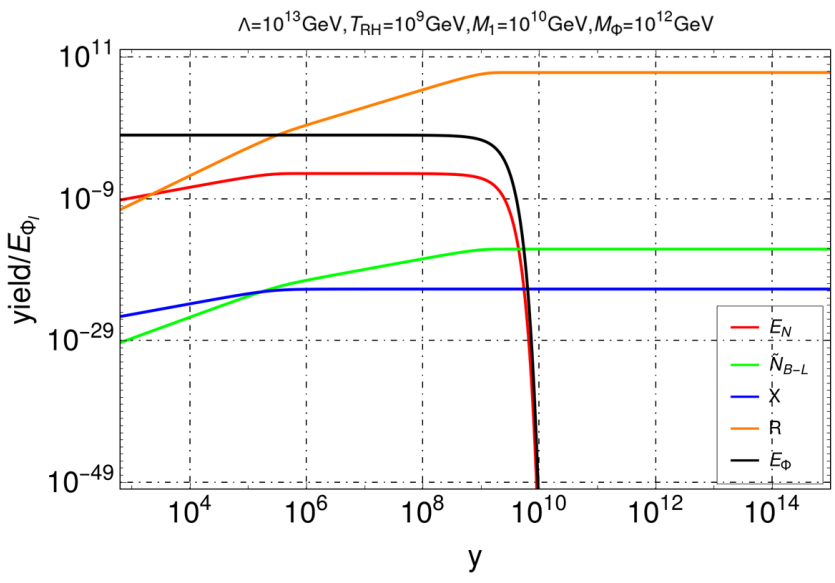

FIG. 6. Yield of different components as a function of $y=$ $a / a_{I}$ is shown for a fixed $\Lambda=10^{13} \mathrm{GeV}, T_{\mathrm{RH}}=10^{9} \mathrm{GeV}$, $M_{\varphi}=10^{12} \mathrm{GeV}$, and $M_{1}=10^{10} \mathrm{GeV}$. The yields corresponding to $\tilde{N}_{B-L}$ and $X$ produce observed baryon asymmetry and DM abundance at $y \rightarrow \infty$ for a DM mass of $10 \mathrm{GeV}$.

curve) produces the right relic abundance. Also, an observed baryon asymmetry is obtained corresponding to $\tilde{N}_{B-L}(y \rightarrow \infty)$. We see, the yield of the different components, except for the DM, follow the pattern of dim.5 interaction as we have seen in Fig. 3. The DM yield flattens and becomes constant at a very early time; $y \sim 10^{5}$. This happens at the same time where the decay of the RHN becomes comparable to its production as we can see comparing the red curve with the blue one. Since the DM yield depends on the pair annihilation of the heavy neutrinos, hence the moment the heavy neutrino decay starts becoming comparable to its production, the DM abundance freezes in and becomes constant. In Fig. 7 we show the evolution of $n_{B-L} / s$ (left) and DM number density (right) with $y=a / a_{I}$. In the left panel we show the variation of $n_{B-L} / s$ for some benchmark choices of $M_{1}$. Here we see $n_{B-L} / s$ evolves in the same way as we have already seen for the dim.5 case in the top left panel of Fig. 4. A more interesting dynamics however appears for the case of DM number density evolution as shown in the right panel. Here we see that the DM number density sharply increases at $y \sim 10$ which falls afterwards and becomes saturated at $y \sim 10^{9}$. The sharp increase of DM number density at very high temperatures is a consequence of heavy neutrino annihilation into DM. As the heavy neutrino starts decaying, the DM number density rapidly falls due to entropy injection in the thermal bath, finally reaching a constant at $y \sim 10^{9}$. The $B-L$ asymmetry also saturates at around the same epoch. This happens because at dim.6 the heavy neutrino becomes the common origin of $B-L$ asymmetry as well as DM genesis and hence the $B-L$ and DM yield build up in a similar fashion. Since at dim.6 the DM abundance via UV freeze-in approximately becomes $\Omega_{\chi} h^{2} \propto m_{\chi} T_{\mathrm{RH}}^{3} / \Lambda^{4}$ hence, compared to the dim.5 

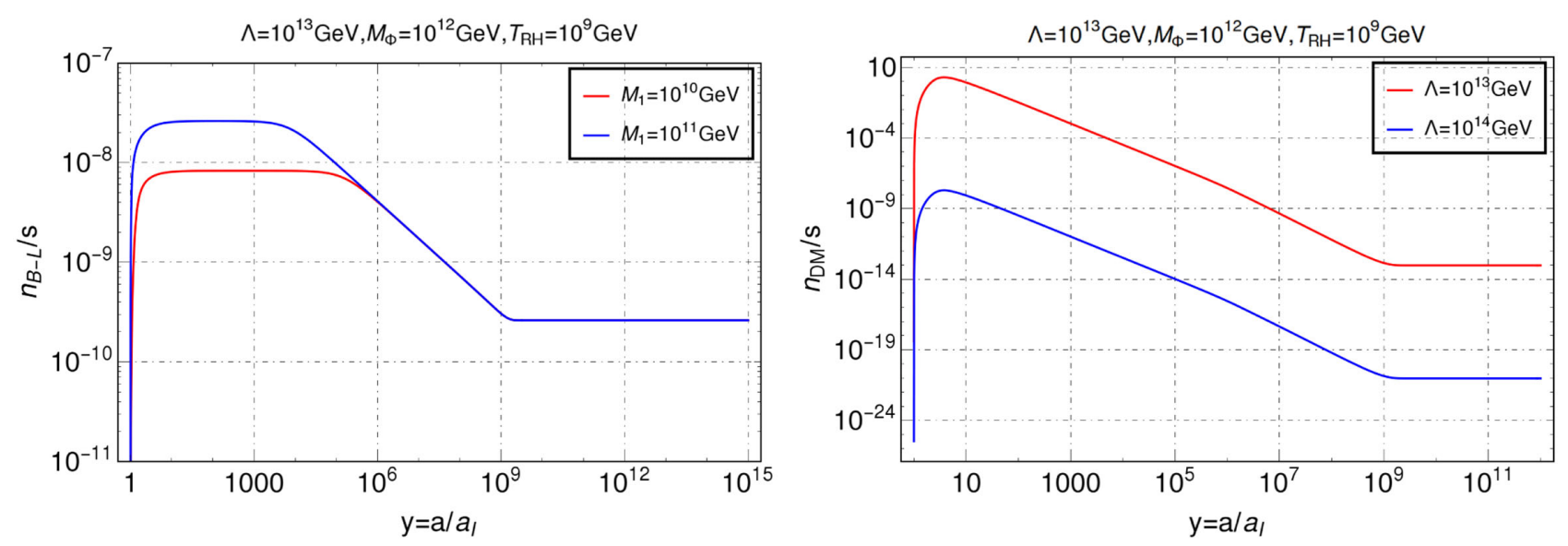

FIG. 7. Left: Evolution of $B-L$ asymmetry with $y=a / a_{I}$ for a fixed inflaton mass $M_{\varphi}=10^{12} \mathrm{GeV}$ and reheat temperature $T_{\mathrm{RH}}=10^{9} \mathrm{GeV}$ for two choices of $M_{1}:\left\{10^{10}, 10^{11}\right\} \mathrm{GeV}$ shown in red and blue respectively. Right: DM yield $n_{\mathrm{DM}} / s$ as a function of $y=a / a_{I}$ for different choices of $\Lambda=\left\{10^{13}, 10^{14}\right\} \mathrm{GeV}$ and $T_{\mathrm{RH}}=10^{9} \mathrm{GeV}$.

case, for a much lower $\Lambda$ observed DM abundance can be achieved for a given DM mass and reheat temperature. We find that for a DM mass of $10 \mathrm{GeV}$ (in order to satisfy the observed relic abundance) one can have $\Lambda=10^{13} \mathrm{GeV}$ for $T_{\mathrm{RH}}=10^{9} \mathrm{GeV}$ which is at least four orders of magnitude smaller than the dim.5 case for a DM mass of the same order. This also establishes our earlier claim that dim. 6 interactions become ineffective in the presence of dim.5 operators.

\section{B. Noninstantaneous inflaton decay}

In case where the instantaneous inflaton decay approximation breaks down, one can no more make the assumption $\Gamma_{\varphi}=\mathcal{H}$ to parametrize the inflaton-heavy neutrino Yukawa coupling in terms of the reheat temperature $T_{\mathrm{RH}}$. In this case the inflaton-heavy neutrino coupling becomes a free parameter. The reheat temperature, on the other hand, can be obtained by defining the temperature at which $E_{\Phi}\left(y_{\mathrm{RH}}\right)=R\left(y_{\mathrm{RH}}\right)$ where $y_{\mathrm{RH}}$ defines the corresponding scale factor ratio. ${ }^{10}$ Similarly, the definition of the reheating temperature for such noninstantaneous inflaton decay scenario has also been used in earlier works, for example [78]. In fact, we have checked, by taking larger $R / E_{\Phi}$ ratio at $y=y_{\mathrm{RH}}$, there is only a slight change in $T_{\mathrm{RH}}$ (that can be neglected). Thus, we stick to the conventional definition already used in earlier works. We first make an estimation of the inflaton-heavy neutrino Yukawa coupling $y_{\varphi}$ and establish a relation between $y_{\varphi}$ and $T_{\mathrm{RH}}$ for dim.5 when the instantaneous decay is no longer an approximation. In the top right panel of Fig. 8 we show the dependence of the

\footnotetext{
${ }^{10}$ For the noninstantaneous case $T_{\mathrm{RH}}$ is defined to be the temperature of the thermal bath when it starts to dominate over the residual energy of the inflaton. This is the point when the energy density of the inflaton starts transferring into the energy density of radiation $[78,102,105]$.
}

Yukawa coupling $y_{\varphi}$ on the reheat temperature during instantaneous decay (in black) and noninstantaneous decay (red dashed) of the inflaton field. In the noninstantaneous decay limit the coupling $y_{\varphi}$ becomes a free parameter, independent of the reheating temperature unlike the instantaneous decay limit where $T_{\mathrm{RH}}$ determines $y_{\varphi}$. Thus, under the noninstantaneous decay approximation one can choose a much lower $y_{\varphi}$ with respect to the instantaneous case (corresponding to a given $T_{\mathrm{RH}}$ ) as evident from the top right panel of Fig. 8. However, for large $y_{\varphi}$ the two limiting cases converge as the decay always happens instantaneously. We thus consider

$$
\left\{\Lambda, y_{\varphi}, m_{\chi}\right\}
$$

to be the relevant free parameters in the context of the noninstantaneous decay scenario. In the top left panel of Fig. 8 we show the evolution of $E_{\varphi}$ and $R$ with $y=a / a_{I}$ for different choices of $y_{\varphi}$ (shown by different colors). For a fixed $y_{\varphi}$ all the solid curves show the evolution of $E_{\varphi}$ while the dashed curves show how the radiation $R$ builds up. By applying the condition $E_{\varphi}=R$ (at each $y=y_{\mathrm{RH}}$ ) we determine $T_{\mathrm{RH}}$ corresponding to each $y_{\varphi}$ which is shown by the red dashed lines in the top right panel. From the top right panel it is also clear that at very high values of $T_{\mathrm{RH}}$ the noninstantaneous approximation merges with the instantaneous one as large $T_{\mathrm{RH}}$ becomes equivalent to large $y_{\varphi}$. One has to, however, be careful about the fact that for a given $M_{\varphi}$, and $M_{1}$ arbitrarily large, the Yukawa coupling (even if within the perturbative limit) needs to be avoided as that might correspond to a reheat temperature $T_{\mathrm{RH}}>M_{1}$ contrary to the requirement for nonthermal leptogenesis. It is also important to note that changing $\Lambda$ while keeping everything else fixed does not change $y_{\mathrm{RH}}$. Therefore, the top right panel plot remains unaltered irrespective of the choices of the cut off scale. We now show the yields 

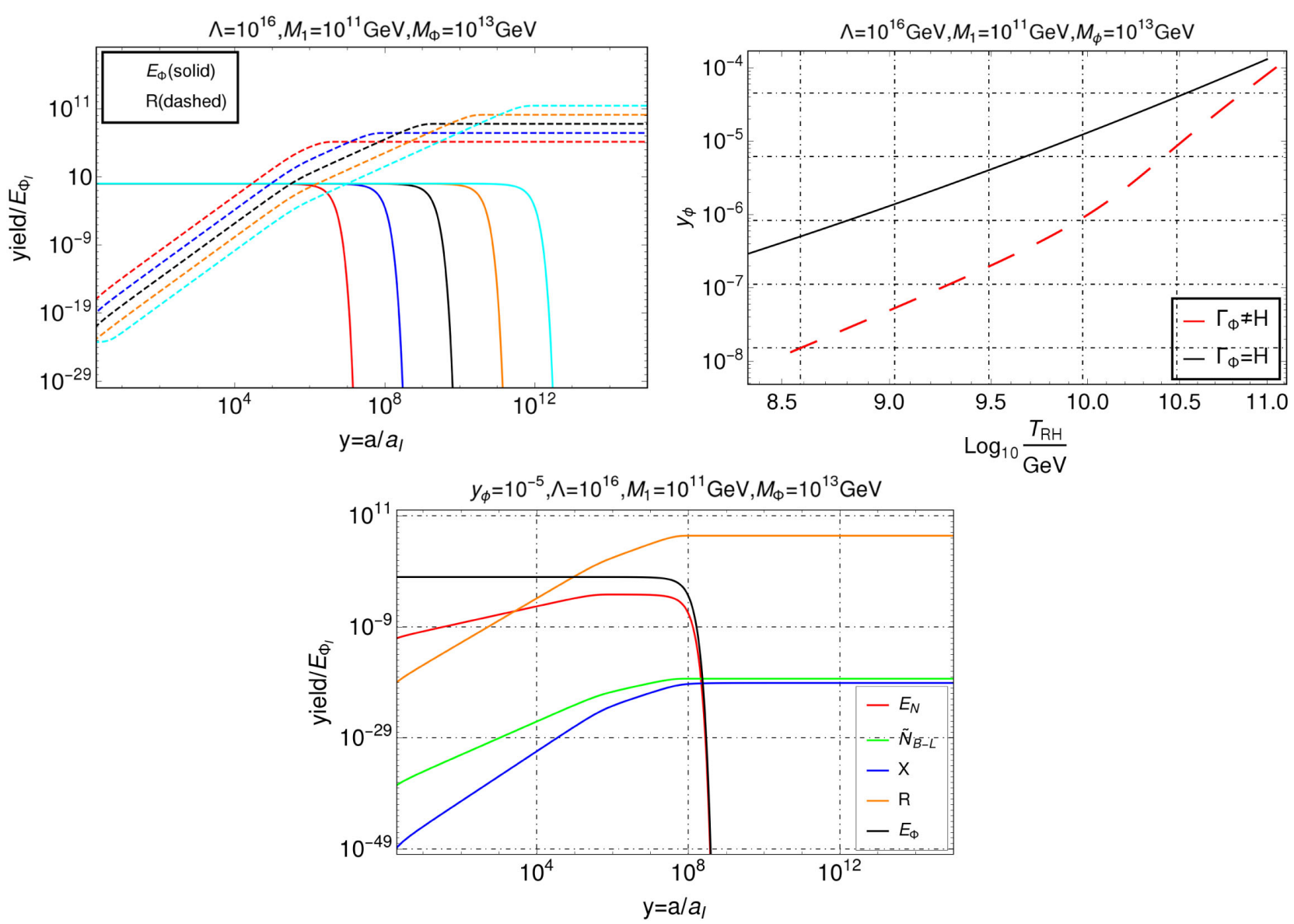

FIG. 8. Top Left: Evolution of $R$ and $E_{\varphi}$ with $y=a / a_{I}$. Here all the solid lines correspond to the variation of $E_{\varphi}$ while all the dashed lines correspond to $R$. Different colored lines indicate different choices of $y_{\varphi}:\left\{10^{-4}, 10^{-5}, 10^{-6}, 10^{-7}, 10^{-8}\right\}$ shown in red, blue, black, orange, and cyan, respectively. The cut off scale, inflaton mass and heavy neutrino mass are fixed at the values mentioned in the plot label. Top Right: A comparison of instantaneous vs noninstantaneous decay approximation in $y_{\varphi}-T_{\mathrm{RH}}$ plane for a fixed choice of other parameters as in the left plot. The reheat temperature for the noninstantaneous case has been obtained from the LHS plot. Bottom: Variation of yield of different components with $y=a / a_{I}$ for fixed values of $y_{\varphi}, \Lambda, M_{\varphi}$ and $M_{1}$ that gives rise to the observed baryon asymmetry and correct relic density for a DM of mass $1 \mathrm{GeV}$.

of the different components as before with $y=a / a_{I}$ in the bottom panel. The nature of the yields remain the same as one can see by comparing with Fig. 3. For $y_{\varphi}=10^{-5}$ and $\Lambda=10^{17} \mathrm{GeV}$ we can obtain the observed DM abundance for a DM of mass $m_{\chi}=1 \mathrm{GeV}$ and baryon asymmetry of the correct order in the limit $y \rightarrow \infty$.

The variation of $n_{B-L} / s$ with $y=a / a_{I}$ for the noninstantaneous scenario is shown in the top left panel of Fig. 9 where we see a larger $M_{1}$ leads to a larger asymmetry for a given $y_{\varphi}$ following the earlier trend in Fig. 4. On the top right panel of Fig. 9 we show how the DM yield $n_{\mathrm{DM}} / s$ evolves with $y=a / a_{I}$ for the same choice of $M_{\varphi}$ and $y_{\varphi}$ as in the top left panel. Here we see the same trend as in Fig. 4 viz a larger $\Lambda$ leads to smaller final DM abundance for a fixed $y_{\varphi}$. This is again same as in the top right panel of Fig. 4 where $T_{\mathrm{RH}}$ plays the same role as $y_{\varphi}$. Thus, for a given DM mass, a larger $\Lambda$ requires a smaller $y_{\varphi}$ to produce the observed relic abundance. In the bottom left panel of Fig. 9 we show the the region of parameter space where the Planck observed relic density is satisfied for DM mass ranging $m_{\chi}:\{1-100\} \mathrm{GeV}$. The inflaton-heavy neutrino Yukawa $y_{\varphi}$, which is now a free parameter, is varied in the range $y_{\varphi}:\left\{10^{-8}-10^{-4}\right\}$. Here we again see, similar to the bottom left panel of Fig. 4, a larger DM mass needs comparatively larger $\Lambda$ to satisfy the observed DM abundance. Since in the case of noninstantaneous inflaton decay, as discussed earlier, a larger $y_{\varphi}$ indicates a larger reheat temperature; hence for a fixed $\Lambda$ increasing $y_{\varphi}$ results in DM over-abundance. Thus, a large $y_{\varphi}$ at a fixed $\Lambda$ requires a smaller DM mass to produce observed DM abundance. Again, since $\Gamma_{\varphi} \propto y_{\varphi}^{2}$, increasing $y_{\varphi}$ increases $N_{1}$ abundance (as $E_{N}^{\prime} \sim \Gamma_{\varphi}$ ) which in turn results in excess in the baryon asymmetry. This can exactly be compared with the effect of increasing $T_{\mathrm{RH}}$ in case of instantaneous decay 

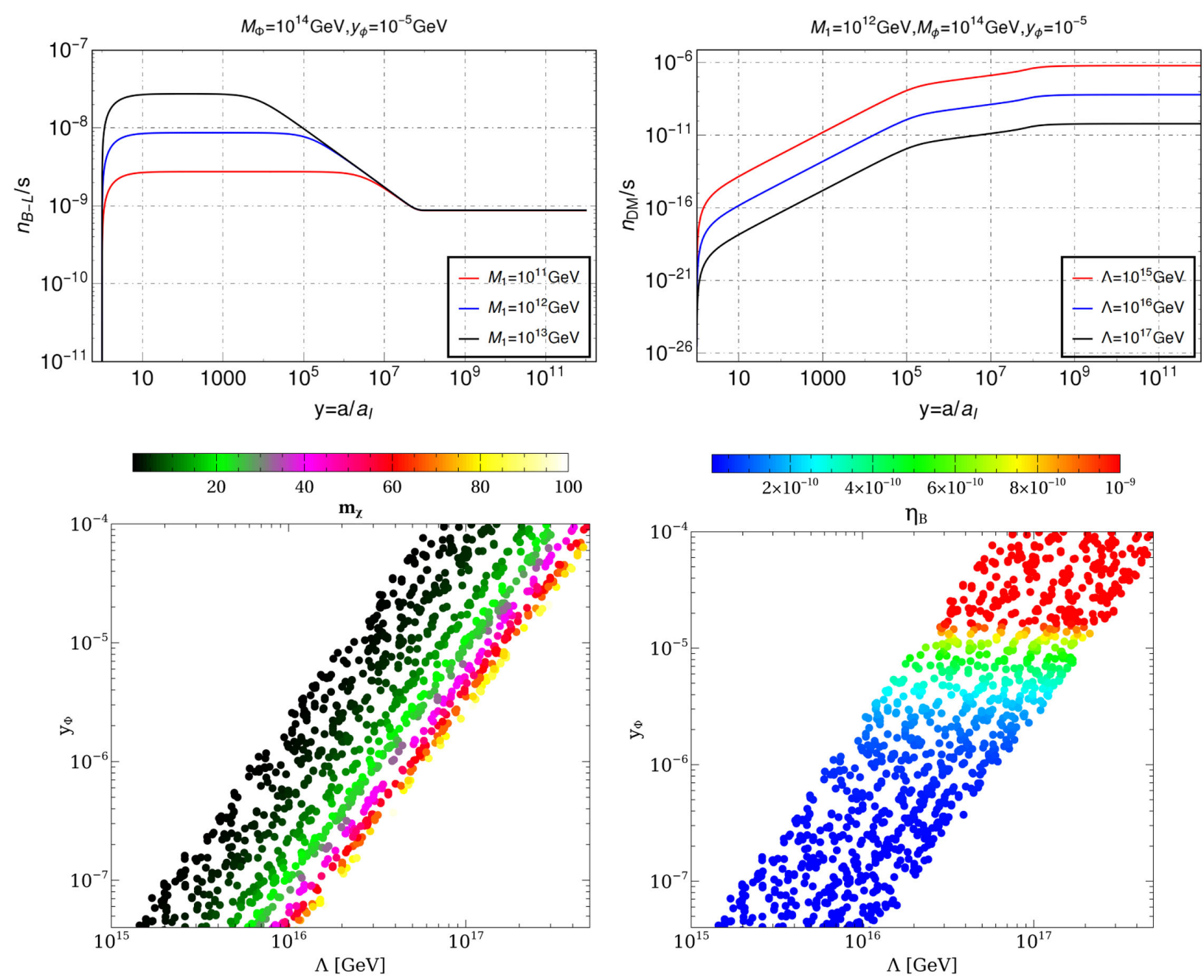

FIG. 9. Top Left: $n_{B-L} / s$ as a function of $y=a / a_{I}$ is shown for a fixed $y_{\varphi}=10^{-5}, M_{\varphi}=10^{13} \mathrm{GeV}$ and for three different choices of $M_{1}:\left\{10^{10}, 10^{11}, 10^{12}\right\} \mathrm{GeV}$ in red, blue, and black respectively. Top Right: Variation of $n_{\mathrm{DM}} / s$ with $y=a / a_{I}$ for a fixed DM mass and different choices of $\Lambda=\left\{10^{15}, 10^{16}, 10^{17}\right\} \mathrm{GeV}$ shown in red, blue, and black respectively. Bottom Left: Relic density allowed parameter space in $y_{\varphi}-\Lambda$ plane where the coloring is with respect to DM mass. Bottom Right: Variation of baryon asymmetry $\eta_{B}$ in $y_{\varphi}-\Lambda$ plane. In both cases the inflaton and heavy neutrino masses are fixed at $M_{\varphi}=10^{13} \mathrm{GeV}$ and $M_{1}=10^{11} \mathrm{GeV}$ respectively.

scenario. The observed asymmetry can be obtained for $y_{\varphi} \sim 10^{-5}$ which certainly produces the right amount of $\mathrm{DM}$ as seen in the bottom left panel. For the case of dim.6, as one can understand, it is possible to obtain the observed DM abundance for a smaller $\Lambda$ as we mentioned earlier in the case of instantaneous decay approximation. Apart from that, other conclusions remain same as the instantaneous decay case and hence we do not elaborate them further.

\section{POSSIBLE UV COMPLETION}

We have realized the possibility of nonthermal leptogenesis and UV freeze-in for a singlet fermionic DM with the inflaton field playing a nontrivial role. Since we are considering hierarchical right-handed neutrinos, the scale of leptogenesis is high; $M_{1}>10^{9} \mathrm{GeV}$. As we have found, to realize the freeze-in scenario, the cut off scale needs to be $\Lambda \gtrsim 10^{15} \mathrm{GeV}$, considering dimension five operators connecting DM and SM bilinears. Although, for DM-SM operators with dimension $d>5$ one can realize UV freezein at a lower cut off scale, the requirement of keeping it above the scale of leptogenesis forces us to consider $\Lambda>$ $M_{1}$ such that the effective theory remains valid. While we stick to this minimal setup to justify UV freeze-in, where the $\mathrm{DM}$ is required to have nonrenormalizable interaction with the SM, such operators can well be realized in a UV complete theory by incorporating additional degrees of freedom. If such additional d.o.f.s have mass $M$, we can generate effective DM-SM operators at a scale $\mu \ll M$, by integrating out those heavy fields. For example, the dim.5 operator $(\bar{\chi} \chi)\left(H^{\dagger} H\right) / \Lambda$ can be generated by extending the model with additional vector like lepton doublet $\psi_{L, R}$. We 
also need $\psi_{L, R}$ to be odd under the discrete $Z_{2}$ symmetry, such that Yukawa interactions of the type

$$
-\mathcal{L} \supset m_{\psi} \bar{\psi} \psi+y_{1} \overline{\psi_{L}} \tilde{H} \chi+y_{2} \overline{\psi_{R}^{c}} H \chi+\text { H.c., }
$$

can be generated. At a scale $\mu \ll m_{\psi}$, the heavy vectorlike leptons can be integrated out, resulting in an operator $y_{1} y_{2}(\bar{\chi} \chi)\left(H^{\dagger} H\right) / \Lambda$, where $\Lambda$ can be identified with the masses of heavy fermions which are integrated out. Note that, by introducing a heavy singlet scalar $\phi$, even under $Z_{2}$, one can have an interaction of the form

$$
-\mathcal{L} \supset y_{N} \bar{N} N \phi+y_{\chi} \bar{\chi} \chi \phi+\text { H.c. }
$$

that can lead to $\operatorname{dim} .6$ operators $\bar{N} N \bar{\chi} \chi / \Lambda^{2}$ on integrating out $\phi$ at a scale $\mu \ll \mu_{\phi}$ with $\Lambda \equiv \mu_{\phi}$. In fact, a heavy $Z_{2}$ even scalar can also provide dim.5 operators of the form $\bar{\chi} \chi H^{\dagger} H$ at a scale $\mu \ll \mu_{\phi}$. While we do not pursue the details of such UV completions, one may refer to several earlier works in this direction. For example, the authors of [58] considered loop suppressions as the origin of FIMP interactions, the possibility of DM-SM interactions via superheavy neutral gauge bosons were discussed in $[106,107]$, while the authors of $[108,109]$ considered clockwork origin of FIMP couplings.

\section{CONCLUSION}

In this work we have addressed two long-standing problems in particle cosmology, namely the matterantimatter asymmetry and the dark matter relic abundance in a minimal setup while keeping the DM analysis model independent. In order to explain both we have considered the SM effective theory being augmented by two righthanded neutrinos $N_{i}$ leading to what is known as $\nu$ SMEFT which explains light neutrino masses via the Type-I seesaw mechanism. We further consider a SM gauge singlet $Z_{2}$ odd fermion that is absolutely stable over the age of the Universe and hence plays the role of a potential DM candidate. While RHNs, in spite of being gauge singlets, can interact with the SM due to the presence of tree-level Yukawa couplings, the $Z_{2}$ odd fermion singlet DM can communicate with the SM only via nonrenormalizable interactions with dimension $d>4$. We consider a high scale Type-I seesaw which favors leptogenesis with hierarchical RHNs. Therefore, we assume new physics at a scale $\Lambda \gg \mathrm{TeV}$, which is also the mass scale of the heavy fields integrated out and write down the operators below $\Lambda$. In the absence of extra fields, the RHN-DM interaction also arises due to four-fermion operators that appear only in dimension six or higher. We take up only scalar DM bilinears for simplicity and thus in dim.6 no SM gauge invariant operator appears connecting the DM with the SM field. At dim.5 level, however, DM-SM interactions can arise involving SM Higgs. Since we focus on nonthermal
DM as well as nonthermal leptogenesis, we consider a singlet scalar field mimicking the inflaton field after slow roll whose decay into RHNs play a crucial role in DM production, leptogenesis as well as overall reheating of the Universe.

We consider baryogenesis via nonthermal leptogenesis where the heavy neutrino masses are larger than the reheat temperature of the Universe for explaining the observed baryon asymmetry. The inflaton exclusively decays into a pair of lightest of the heavy neutrinos $N_{1}$ via a renormalizable interaction. The heavy neutrino $N_{1}$ thus produced further decays into SM bath producing a net $B-L$ asymmetry that is converted into a baryon asymmetry by sphaleron processes. The decay of the inflaton field into $N_{1}$ pair can be either instantaneous or noninstantaneous where in the former case the inflaton-RHN coupling is parametrized by the reheat temperature, while in the latter the coupling can be considered to be a free parameter. We analyse both the cases separately and show it is possible to obtain the observed baryon asymmetry depending on the choice of reheat temperature (for instantaneous decay case) or the inflation-RHN Yukawa coupling (for noninstantaneous decay case) together with the heavy neutrino mass.

The higher dimensional operators mediating DM-SM and DM-RHN interactions motivate us to consider UV freeze-in as the mechanism for DM genesis. At dim.5 level, the DM can get produced from the SM bath itself which is again generated from the out-of-equilibrium decay of $N_{1}$. At dim.6, on the other hand, the only source of DM is the four-fermion operator consisting of the RHN and the DM fields. Since dim.6 interactions become subdominant in presence of dim.5 operators, hence we take up each cases one by one and analyse them separately. Since the inflaton decay triggers the leptogenesis while the DM can be produced both from the SM bath and the heavy neutrinos depending on the dimension of interaction, hence we solve a set of coupled Boltzmann equations to obtain the final yield of different components. Because of the coupled nature of the solutions, a very nontrivial dynamics in the resulting yield shows up. We find that the observed baryon asymmetry together with the right DM abundance can be obtained in dim.5 for $\Lambda \gtrsim 10^{15} \mathrm{GeV}$ for DM mass up to $100 \mathrm{GeV}$ with $T_{\mathrm{RH}} \gtrsim 10^{9} \mathrm{GeV}$. This translates into a bound on inflaton-RHN coupling $10^{-7} \lesssim y_{\varphi} \lesssim 10^{-4}$ for the noninstantaneous reheating scenario. In case of dim.6 interaction (where dim.5 operators are absent) the bound on the cut off scale is significantly lifted up to $\Lambda \gtrsim 10^{14} \mathrm{GeV}$ for the same set of parameters. In all such cases we make sure that the inflaton mass $M_{\varphi}>2 M_{1}$ and $M_{1}<T_{\mathrm{RH}}$ in compliance with our previous assumptions. As the DM in the case of dim.6 interaction is produced from the heavy neutrino annihilation here we see a very intriguing dynamics in the DM yield. The DM yield shows a peak at early times and then gets diluted with the subsequent entropy injection as the heavy neutrino starts decaying. This 
behavior is absent in dim.5 as the DM there gets produced only from the radiation and hence the two, namely the radiation and the DM energy density evolve in a similar way. While we perform a complete numerical analysis by solving the coupled Boltzmann equations, for the sake of completeness, we have also compared our numerical estimation of baryon asymmetry with the one obtained using approximate analytical formula under the assumption of instantaneous RHN decay. Comparing the analytical and numerical results in one of the cases, we found that the overall baryon asymmetry behaves similarly with the reheat temperature under both approaches, although the numerical analysis leads to slightly larger values.

Finally, in presence of heavy neutrinos, inflaton decay alone can provide a common origin of observed baryon asymmetry and DM abundance in the present Universe along with successful reheating. While the role of inflaton field in nonthermal leptogenesis and DM production has been studied separately in earlier works, we have presented an analysis combining both and indicating the interesting interplay of inflaton, right-handed neutrinos and DM leading to the desired phenomenology including light neutrino masses and mixing. This framework, though numerically challenging because of the presence of five coupled Boltzmann equations, is economical in terms of the free parameters. Since we have separated the inflationary or exponential expansion phase from post-inflationary reheating, there certainly remain prospects of connecting such a set-up to more exotic physics or model-dependent scenarios where a specific form of inflationary potentials, both during and after the slow-roll phase can dictate the dynamics of DM and lepton asymmetry yields. We leave such detailed studies for future endeavors.

\section{ACKNOWLEDGMENTS}

D. B. acknowledges the support from Early Career Research Award from DST-SERB, Government of India (Reference No. ECR/2017/001873). R. R. would like to acknowledge fruitful discussion with Qaisar Shafi and Arunansu Sil. B. B. would like to acknowledge the uninterrupted and fast internet service from BSNL (Bharat Sanchar Nigam Limited) which helped the work to proceed smoothly and efficiently.

\section{APPENDIX: MAXIMUM TEMPERATURE DURING REHEATING}

Assuming the inflaton energy density dominates in early times $E_{\varphi_{I}} \gg H_{I}$ we can write from the radiation evolution equation

$$
\left.\frac{d R}{d y}\right|_{\text {early }}=\frac{\Gamma_{N}}{H} E_{N} \approx \frac{\Gamma_{N}}{\sqrt{\frac{16 \pi E_{\varphi_{I}}}{3 M_{\mathrm{pl}}^{2} y^{3}}}} E_{\varphi_{I}}=\Gamma_{N} E_{\varphi_{I}}\left(\frac{3 M_{\mathrm{pl}}^{2}}{16 \pi}\right)^{1 / 2} \frac{y^{3 / 2}}{\sqrt{E_{\varphi_{I}}}},
$$

where $H_{I}$ is determined via

$$
E_{\varphi_{I}}=\frac{3 M_{\mathrm{pl}}^{2}}{8 \pi} H_{I}^{2},
$$

as $E_{\varphi_{I}}=\rho_{\varphi_{I}} a_{I}^{3}$. Now, at $y=1, R=0$ which gives

$$
\begin{aligned}
T= & \left(\frac{30}{\pi^{2} g_{\star}(T)}\right)^{1 / 4}\left(\frac{3}{20 \sqrt{2} \pi}\right)^{1 / 4} \Gamma_{N}^{1 / 4} M_{\mathrm{pl}}^{1 / 2} H_{I}^{1 / 4} \\
& \times\left(y^{-3 / 2}-y^{-4}\right)^{1 / 4} .
\end{aligned}
$$

Thus, we can write

$$
T=T_{\max } \xi(y),
$$

where $\xi(y)=\left(y^{-3 / 2}-y^{-4}\right)^{1 / 4}$ that starts from zero and grows until $y_{\max }=(8 / 3)^{2 / 5}$ where it reaches a maximum $\xi_{\max }=1$ which corresponds to $T=T_{\max }$ and then decreases as $y^{-3 / 8}$. Now, as $\Gamma_{N} \sim M_{1} / 8 \pi>T_{\mathrm{RH}}$, therefore,

$T_{\max }>(8 / 3)^{2 / 5}\left(\frac{30}{\pi^{2} g_{\star}(T)}\right)^{1 / 4}\left(\frac{3}{20 \sqrt{2} \pi}\right)^{1 / 4} M_{1}^{1 / 4} M_{\mathrm{pl}}^{1 / 2} H_{I}^{1 / 4}$.

For a model of chaotic inflation $H_{I} \sim M_{\varphi}$, then

$$
\begin{aligned}
T_{\max } \gtrsim & 10^{14} \mathrm{GeV}\left(\frac{30}{\pi^{2} g_{\star}\left(T_{\max }\right)}\right)^{1 / 4} M_{\mathrm{pl}}^{1 / 2}\left(\frac{H_{I}}{10^{14} \mathrm{GeV}}\right)^{1 / 4} \\
& \times\left(\frac{M_{1}}{10^{12} \mathrm{GeV}}\right)^{1 / 4} .
\end{aligned}
$$

[1] Particle Data Group Collaboration, Review of particle physics, Prog. Theor. Exp. Phys. 2020, 083C01 (2020).
[2] Planck Collaboration, Planck 2018 results. VI. Cosmological parameters, Astron. Astrophys. 641, A6 (2020). 
[3] A. D. Sakharov, Violation of $C P$ invariance, C asymmetry, and baryon asymmetry of the universe, Pis'ma Zh. Eksp. Teor. Fiz. 5, 32 (1967).

[4] S. Weinberg, Cosmological Production of Baryons, Phys. Rev. Lett. 42, 850 (1979).

[5] E. W. Kolb and S. Wolfram, Baryon number generation in the early universe, Nucl. Phys. B172, 224 (1980).

[6] M. Fukugita and T. Yanagida, Baryogenesis without grand unification, Phys. Lett. B 174, 45 (1986).

[7] V. A. Kuzmin, V. A. Rubakov, and M. E. Shaposhnikov, On the anomalous electroweak baryon number nonconservation in the early universe, Phys. Lett. 155B, 36 (1985).

[8] C. S. Fong, E. Nardi, and A. Riotto, Leptogenesis in the Universe, Adv. High Energy Phys. 2012, 158303 (2012).

[9] R. N. Mohapatra and G. Senjanovic, Neutrino Mass and Spontaneous Parity Violation, Phys. Rev. Lett. 44, 912 (1980).

[10] T. Yanagida, Horizontal symmetry and masses of neutrinos, Conf. Proc. C7902131, 95 (1979).

[11] M. Gell-Mann, P. Ramond, and R. Slansky, Complex spinors and unified theories, Conf. Proc. C790927, 315 (1979).

[12] S. L. Glashow, The future of elementary particle physics, NATO Sci. Ser. B 61, 687 (1980).

[13] J. Schechter and J. W. F. Valle, Neutrino masses in $\mathrm{SU}(2) \times \mathrm{U}(1)$ theories, Phys. Rev. D 22, 2227 (1980).

[14] W. Buchmuller, P. Di Bari, and M. Plumacher, Leptogenesis for pedestrians, Ann. Phys. (Amsterdam) 315, 305 (2005).

[15] A. Dutta Banik, R. Roshan, and A. Sil, Neutrino mass and asymmetric dark matter: Study with inert Higgs doublet and high scale validity, J. Cosmol. Astropart. Phys. 03 (2021) 037.

[16] S. Davidson and A. Ibarra, A lower bound on the righthanded neutrino mass from leptogenesis, Phys. Lett. B 535, 25 (2002).

[17] A. Pilaftsis and T. E. J. Underwood, Resonant leptogenesis, Nucl. Phys. B692, 303 (2004).

[18] P. S. B. Dev, M. Garny, J. Klaric, P. Millington, and D. Teresi, Resonant enhancement in leptogenesis, Int. J. Mod. Phys. A 33, 1842003 (2018).

[19] G. Lazarides and Q. Shafi, Origin of matter in the inflationary cosmology, Phys. Lett. B 258, 305 (1991).

[20] H. Murayama, H. Suzuki, T. Yanagida, and J. Yokoyama, Chaotic Inflation and Baryogenesis By Right-Handed Sneutrinos, Phys. Rev. Lett. 70, 1912 (1993).

[21] E. W. Kolb, A. D. Linde, and A. Riotto, GUT Baryogenesis After Preheating, Phys. Rev. Lett. 77, 4290 (1996).

[22] G. Giudice, M. Peloso, A. Riotto, and I. Tkachev, Production of massive fermions at preheating and leptogenesis, J. High Energy Phys. 08 (1999) 014.

[23] T. Asaka, K. Hamaguchi, M. Kawasaki, and T. Yanagida, Leptogenesis in inflaton decay, Phys. Lett. B 464, 12 (1999).

[24] T. Asaka, K. Hamaguchi, M. Kawasaki, and T. Yanagida, Leptogenesis in inflationary universe, Phys. Rev. D 61, 083512 (2000).

[25] K. Hamaguchi, H. Murayama, and T. Yanagida, Leptogenesis from $\mathrm{N}$ dominated early universe, Phys. Rev. D 65, 043512 (2002).
[26] R. Jeannerot, S. Khalil, and G. Lazarides, Leptogenesis in smooth hybrid inflation, Phys. Lett. B 506, 344 (2001).

[27] M. Fujii, K. Hamaguchi, and T. Yanagida, Leptogenesis with almost degenerate Majorana neutrinos, Phys. Rev. D 65, 115012 (2002).

[28] G. Giudice, A. Notari, M. Raidal, A. Riotto, and A. Strumia, Towards a complete theory of thermal leptogenesis in the SM and MSSM, Nucl. Phys. B685, 89 (2004).

[29] S. Pascoli, S. Petcov, and C. Yaguna, Quasidegenerate neutrino mass spectrum, mu- $\varnothing e+$ gamma decay and leptogenesis, Phys. Lett. B 564, 241 (2003).

[30] T. Asaka, H. Nielsen, and Y. Takanishi, Nonthermal leptogenesis from the heavier Majorana neutrinos, Nucl. Phys. B647, 252 (2002).

[31] G. Panotopoulos, Non-thermal leptogenesis and baryon asymmetry in different neutrino mass models, Phys. Lett. B 643, 279 (2006).

[32] F. Hahn-Woernle and M. Plumacher, Effects of reheating on leptogenesis, Nucl. Phys. B806, 68 (2009).

[33] W. Buchmuller, V. Domcke, K. Kamada, and K. Schmitz, A minimal supersymmetric model of particle physics and the early universe, arXiv:1309.7788.

[34] D. Croon, N. Fernandez, D. McKeen, and G. White, Stability, reheating and leptogenesis, J. High Energy Phys. 06 (2019) 098.

[35] D. Borah, S. Jyoti Das, and A. K. Saha, Cosmic inflation in minimal $U(1)_{B-L}$ model: Implications for (Non) thermal dark matter and leptogenesis, Eur. Phys. J. C 81, 169 (2021).

[36] R. Samanta, A. Biswas, and S. Bhattacharya, Non-thermal production of lepton asymmetry and dark matter in minimal seesaw with right-handed neutrino induced Higgs potential, J. Cosmol. Astropart. Phys. 01 (2021) 055.

[37] F. Zwicky, Die Rotverschiebung von extragalaktischen Nebeln, Helv. Phys. Acta 6, 110 (1933).

[38] V. C. Rubin and W. K. Ford, Jr., Rotation of the andromeda nebula from a spectroscopic survey of emission regions, Astrophys. J. 159, 379 (1970).

[39] D. Clowe, M. Bradac, A. H. Gonzalez, M. Markevitch, S. W. Randall, C. Jones, and D. Zaritsky, A direct empirical proof of the existence of dark matter, Astrophys. J. Lett. 648, L109 (2006).

[40] M. Srednicki, R. Watkins, and K. A. Olive, Calculations of relic densities in the early universe, Nucl. Phys. B310, 693 (1988).

[41] P. Gondolo and G. Gelmini, Cosmic abundances of stable particles: Improved analysis, Nucl. Phys. B360, 145 (1991).

[42] E. W. Kolb and M. S. Turner, The early universe, Front. Phys. 69, 1 (1990).

[43] E. Aprile et al., Dark Matter Search Results from a One Tonne $\times$ Year Exposure of XENON1T, Phys. Rev. Lett. 121, 111302 (2018).

[44] L. J. Hall, K. Jedamzik, J. March-Russell, and S. M. West, Freeze-in production of FIMP dark matter, J. High Energy Phys. 03 (2010) 080.

[45] N. Bernal, M. Heikinheimo, T. Tenkanen, K. Tuominen, and V. Vaskonen, The dawn of FIMP dark matter: A review 
of models and constraints, Int. J. Mod. Phys. A 32, 1730023 (2017).

[46] G. Arcadi and L. Covi, Minimal decaying dark matter and the LHC, J. Cosmol. Astropart. Phys. 08 (2013) 005.

[47] C. E. Yaguna, The singlet scalar as FIMP dark matter, J. High Energy Phys. 08 (2011) 060.

[48] X. Chu, T. Hambye, and M. H. G. Tytgat, The four basic ways of creating dark matter through a portal, J. Cosmol. Astropart. Phys. 05 (2012) 034.

[49] M. Blennow, E. Fernandez-Martinez, and B. Zaldivar, Freeze-in through portals, J. Cosmol. Astropart. Phys. 01 (2014) 003.

[50] A. Merle and M. Totzauer, keV sterile neutrino dark matter from singlet scalar decays: Basic concepts and subtle features, J. Cosmol. Astropart. Phys. 06 (2015) 011.

[51] B. Shakya, Sterile neutrino dark matter from freeze-in, Mod. Phys. Lett. A 31, 1630005 (2016).

[52] A. G. Hessler, A. Ibarra, E. Molinaro, and S. Vogl, Probing the scotogenic FIMP at the LHC, J. High Energy Phys. 01 (2017) 100 .

[53] A. Biswas and A. Gupta, Freeze-in production of sterile neutrino dark matter in $\mathrm{U}(1)_{\mathrm{B}-\mathrm{L}}$ model, J. Cosmol. Astropart. Phys. 09 (2016) 044.

[54] J. Knig, A. Merle, and M. Totzauer, keV sterile neutrino dark matter from singlet scalar decays: The most general case, J. Cosmol. Astropart. Phys. 11 (2016) 038.

[55] A. Biswas and A. Gupta, Calculation of momentum distribution function of a non-thermal fermionic dark matter, J. Cosmol. Astropart. Phys. 03 (2017) 033.

[56] A. Biswas, S. Choubey, and S. Khan, FIMP and muon $(g-2)$ in a $\mathrm{U}(1)_{L_{\mu}-L_{\tau}}$ model, J. High Energy Phys. 02 (2017) 123.

[57] M. Duch, B. Grzadkowski, and D. Huang, Strongly selfinteracting vector dark matter via freeze-in, J. High Energy Phys. 01 (2018) 020.

[58] A. Biswas, D. Borah, and A. Dasgupta, UV complete framework of freeze-in massive particle dark matter, Phys. Rev. D 99, 015033 (2019).

[59] S. Heeba, F. Kahlhoefer, and P. Stcker, Freeze-in production of decaying dark matter in five steps, J. Cosmol. Astropart. Phys. 11 (2018) 048.

[60] S. Peyman Zakeri, S. Mohammad Moosavi Nejad, M. Zakeri, and S. Yaser Ayazi, A minimal model for twocomponent FIMP dark matter: A basic search, Chin. Phys. C 42, 073101 (2018).

[61] M. Becker, Dark matter from freeze-in via the neutrino portal, Eur. Phys. J. C 79, 611 (2019).

[62] S. Heeba and F. Kahlhoefer, Probing the freeze-in mechanism in dark matter models with U(1) gauge extensions, Phys. Rev. D 101, 035043 (2020).

[63] O. Lebedev and T. Toma, Relativistic freeze-in, Phys. Lett. B 798, 134961 (2019).

[64] B. Barman, S. Bhattacharya, and M. Zakeri, Non-abelian vector boson as FIMP dark matter, J. Cosmol. Astropart. Phys. 02 (2020) 029.

[65] S. Bhattacharya, N. Chakrabarty, R. Roshan, and A. Sil, Multicomponent dark matter in extended $U(1)_{B-L}$ : Neutrino mass and high scale validity, J. Cosmol. Astropart. Phys. 04 (2020) 013.
[66] A. Datta, R. Roshan, and A. Sil, Imprint of the seesaw mechanism on feebly interacting dark matter and the baryon asymmetry, arXiv:2104.02030.

[67] S. Bhattacharya, R. Roshan, A. Sil, and D. Vatsyayan, Symmetry origin of baryon asymmetry, dark matter and neutrino mass, arXiv:2105.06189.

[68] F. Elahi, C. Kolda, and J. Unwin, Ultraviolet freeze-in, J. High Energy Phys. 03 (2015) 048.

[69] J. McDonald, Warm dark matter via ultra-violet freeze-in: Reheating temperature and non-thermal distribution for fermionic Higgs portal dark matter, J. Cosmol. Astropart. Phys. 08 (2016) 035.

[70] S.-L. Chen and Z. Kang, On ultraviolet freeze-in dark matter during reheating, J. Cosmol. Astropart. Phys. 05 (2018) 036.

[71] A. Biswas, S. Ganguly, and S. Roy, Fermionic dark matter via UV and IR freeze-in and its possible X-ray signature, J. Cosmol. Astropart. Phys. 03 (2020) 043.

[72] N. Bernal, F. Elahi, C. Maldonado, and J. Unwin, Ultraviolet freeze-in and non-standard cosmologies, J. Cosmol. Astropart. Phys. 11 (2019) 026.

[73] N. Bernal, J. Rubio, and H. Veerme, Boosting ultraviolet freeze-in in NO models, J. Cosmol. Astropart. Phys. 06 (2020) 047.

[74] N. Bernal, J. Rubio, and H. Veermäe, UV freeze-in in Starobinsky inflation, J. Cosmol. Astropart. Phys. 10 (2020) 021.

[75] B. Barman, D. Borah, and R. Roshan, Effective theory of freeze-in dark matter, J. Cosmol. Astropart. Phys. 11 (2020) 021.

[76] B. Barman, S. Bhattacharya, and B. Grzadkowski, Feebly coupled vector boson dark matter in effective theory, J. High Energy Phys. 12 (2020) 162.

[77] B. Barman, A. Dutta Banik, and A. Paul, Implications of NANOGrav results and UV freeze-in in a fast-expanding Universe, arXiv:2012.11969.

[78] M. A. G. Garcia, K. Kaneta, Y. Mambrini, and K. A. Olive, Reheating and post-inflationary production of dark matter, Phys. Rev. D 101, 123507 (2020).

[79] F. del Aguila, S. Bar-Shalom, A. Soni, and J. Wudka, Heavy Majorana neutrinos in the effective lagrangian description: Application to hadron colliders, Phys. Lett. B 670, 399 (2009).

[80] A. Aparici, K. Kim, A. Santamaria, and J. Wudka, Righthanded neutrino magnetic moments, Phys. Rev. D 80, 013010 (2009).

[81] S. Bhattacharya and J. Wudka, Dimension-seven operators in the standard model with right handed neutrinos, Phys. Rev. D 94, 055022 (2016).

[82] Y. Liao and X.-D. Ma, Operators up to dimension seven in Standard Model effective field theory extended with sterile neutrinos, Phys. Rev. D 96, 015012 (2017).

[83] M. Chala and A. Titov, One-loop matching in the SMEFT extended with a sterile neutrino, J. High Energy Phys. 05 (2020) 139.

[84] I. Bischer, T. Plehn, and W. Rodejohann, Dark matter EFT, the third-neutrino WIMPs, SciPost Phys. 10, 039 (2021).

[85] W. Buchmuller, R. D. Peccei, and T. Yanagida, Leptogenesis as the origin of matter, Annu. Rev. Nucl. Part. Sci. 55, 311 (2005). 
[86] M. Kawasaki, K. Kohri, and T. Moroi, Big-Bang nucleosynthesis and hadronic decay of long-lived massive particles, Phys. Rev. D 71, 083502 (2005).

[87] V. Senoguz and Q. Shafi, Gut scale inflation, non-thermal leptogenesis, and atmospheric neutrino oscillations, Phys. Lett. B 582, 6 (2004).

[88] K. Kaneta, Y. Mambrini, and K. A. Olive, Radiative production of nonthermal dark matter, Phys. Rev. D 99, 063508 (2019).

[89] P. Minkowski, $e$ at a rate of one out of 109 muon decays?, Phys. Lett. 67B, 421 (1977).

[90] J. A. Casas and A. Ibarra, Oscillating neutrinos and $\mu \rightarrow e, \gamma$, Nucl. Phys. B618, 171 (2001).

[91] S. Davidson, E. Nardi, and Y. Nir, Leptogenesis, Phys. Rep. 466, 105 (2008).

[92] J. Edsjo and P. Gondolo, Neutralino relic density including coannihilations, Phys. Rev. D 56, 1879 (1997).

[93] T. Hasegawa, N. Hiroshima, K. Kohri, R. S. L. Hansen, T. Tram, and S. Hannestad, MeV-scale reheating temperature and thermalization of oscillating neutrinos by radiative and hadronic decays of massive particles, J. Cosmol. Astropart. Phys. 12 (2019) 012.

[94] T. Moroi, H. Murayama, and M. Yamaguchi, Cosmological constraints on the light stable gravitino, Phys. Lett. B 303, 289 (1993).

[95] M. Kawasaki and T. Moroi, Gravitino production in the inflationary universe and the effects on big bang nucleosynthesis, Prog. Theor. Phys. 93, 879 (1995).

[96] L. Kofman, A. D. Linde, and A. A. Starobinsky, Towards the theory of reheating after inflation, Phys. Rev. D 56, 3258 (1997).

[97] A. D. Linde, Particle physics and inflationary cosmology, Contemp. Concepts Phys. 5, 1 (1990).

[98] Y. Ema, R. Jinno, K. Mukaida, and K. Nakayama, Gravitational particle production in oscillating backgrounds and its cosmological implications, Phys. Rev. D 94, 063517 (2016).

[99] Y. Ema, K. Nakayama, and Y. Tang, Production of purely gravitational dark matter, J. High Energy Phys. 09 (2018) 135.

[100] Y. Mambrini and K. A. Olive, Gravitational production of dark matter during reheating, Phys. Rev. D 103, 115009 (2021).

[101] B. Barman and N. Bernal, Gravitational SIMPs, arXiv: 2104.10699.

[102] M. A. G. Garcia, Y. Mambrini, K. A. Olive, and M. Peloso, Enhancement of the Dark matter abundance before reheating: Applications to gravitino dark matter, Phys. Rev. D 96, 103510 (2017).

[103] M. Chianese and S. F. King, The dark side of the littlest seesaw: Freeze-in, the two right-handed neutrino portal and leptogenesis-friendly fimpzillas, J. Cosmol. Astropart. Phys. 09 (2018) 027.

[104] M. Chianese, B. Fu, and S. F. King, Minimal seesaw extension for neutrino mass and mixing, leptogenesis and dark matter: FIMPzillas through the right-handed neutrino portal, J. Cosmol. Astropart. Phys. 03 (2020) 030.

[105] S. Davidson and S. Sarkar, Thermalization after inflation, J. High Energy Phys. 11 (2000) 012.

[106] Y. Mambrini, K. A. Olive, J. Quevillon, and B. Zaldivar, Gauge Coupling Unification and Nonequilibrium Thermal Dark Matter, Phys. Rev. Lett. 110, 241306 (2013).

[107] G. Bhattacharyya, M. Dutra, Y. Mambrini, and M. Pierre, Freezing-in dark matter through a heavy invisible Z, Phys. Rev. D 98, 035038 (2018).

[108] J. Kim and J. McDonald, Clockwork Higgs portal model for freeze-in dark matter, Phys. Rev. D 98, 023533 (2018).

[109] J. Kim and J. Mcdonald, Freeze-in dark matter from a subHiggs mass clockwork sector via the Higgs portal, Phys. Rev. D 98, 123503 (2018). 